\title{
Cleat-scale modelling of the coal permeability evolution due to sorption-induced strain
}

\author{
François BERTRAND ${ }^{\mathrm{a}, \mathrm{b}, \mathrm{c}}$, Olivier BUZZI ${ }^{\mathrm{b}}$, Frédéric COLLIN $^{\mathrm{a}}$ \\ ${ }^{a}$ University of Liège, Urban and Environmental Engineering / Geomechanics, Liège, Belgium \\ ${ }^{b}$ University of Newcastle, Priority Research Centre for Geotechnical and Materials Modelling, Callaghan, \\ NSW, Australia \\ ${ }^{c}$ F.R.I.A, Fonds de la Recherche Scientifique - FNRS, Brussels, Belgium
}

\begin{abstract}
Coal permeability is known to be affected by the sorption-induced strain. Indeed, coal swells with gas sorption and shrinks with desorption, which is likely to modify the cleat aperture and thus the permeability. Coal permeability evolution is crucially important for either Coalbed Methane production (CBM) or Carbon dioxide Capture and Storage (CCS).

A model is developed at the scale of the fractures to take into account the hydromechanical couplings observed experimentally. It is implemented in the finite element code Lagamine. This numerical model is developed at the scale of the fractures and the matrix blocks. Fractures are modelled with interface elements specially adapted to manage sorption/desorption by taking into account the Langmuir's isotherm (Langmuir, 1918). In the matrix blocks, the sorption strain is assumed proportional to the adsorbed gas content. Depending on the boundary conditions, sorption strain affects the stress state and thus the fracture aperture. Contrary to macroscale models (Bertrand et al., 2017), this model does not require the use of shape factors since the geometry can be explicitly represented. The implementation of the model is validated by comparison with the analytical solution on a simple geometry. The model is finally used to simulate gas injection on a representative elementary volume (REV) made up few matrix blocks. This modelling highlights the interests of a numerical approach to compute the permeability evolution compared to a porosity-based model.
\end{abstract}

Keywords: Numerical model, Analytical model, Permeability, Fractured medium, Couplings, Swelling, Langmuir

\section{Introduction}

Coal seams generally contain large amounts of methane that can be recovered in the form of natural gas, the so-called coalbed methane (CBM). This gas is mainly trapped by sorption thanks to the high internal surface of the coal matrix, ranging from 20 to $300 \mathrm{~m}^{2} / \mathrm{g}$ (Berkowitz, 1985). Un-mined coal seams are also interesting reservoirs in the context of Carbon dioxide Capture and Storage (CCS) (White et al., 2005) in order 
to mitigate the climate change (Intergovernmental Panel on Climate Change - IPCC, 2015).

Coals are naturally fractured reservoirs and the fractures, termed cleats, are preferential pathways for the methane production or carbon storage. The permeability of the reservoir is mainly function of the aperture and the density of the cleats. Estimates of cleat aperture under in situ confining stress vary from 0.1 to $0.0001 \mathrm{~mm}$ (Harpalani and Chen, 1997). Any change of the cleat network likely modifies the permeability of the material. During gas production, the permeability is sensitive to two distinct mechanisms (Gray et al., 1987): the reservoir compaction due to the increase of effective stress following the depletion of the reservoir, and the matrix shrinkage due to the gas desorption. The reservoir permeability depends therefore on the net influence of these two competing phenomena. Reversely, the injection of carbon dioxide leads to a complex interaction with the matrix which involves coal swelling and is likely to affect the permeability.

Many models taking into account the coal permeability evolution with the gas pressure have been developed over the past few decades (Somerton et al., 1975; Schwerer et al., 1984; Seidle et al., 1992; Gilman and Beckie, 2000; Pan and Connell, 2007, 2012; Shi et al., 2014; Peng et al., 2017; Bertrand et al., 2017). However, these models are either analytical formulations or macroscopic models. In this work, a hydro-mechanical model is formulated at the scale of the coal constituents, i.e. cleats and matrix blocks, and then implemented into the numerical code Lagamine developed by the University of Liège (Charlier, 1987; Habraken, 1989). At this scale, a channel flow model is directly derived from Navier-Stokes equations and cleats are modelled thanks to interface elements. It means the permeability evolution is not based on a porosity model and the use of shape factor is not required since the geometry is explicitly represented.

In the following, the hydro-mechanical model for both the matrix and the cleats is presented in details. The sorption isotherm is included in the formulation of the gas transfer between the matrix and the cleats to account for the change of state. The implementation in the finite element code is briefly presented with a special attention paid to the interface elements. Then, the implementation of the model is validated by comparison with analytical models. Finally, the numerical model is used for the modelling on a representative elementary volume. The permeability alteration due to $\mathrm{CO}_{2}$ is modelled for different boundary conditions.

\section{Hydro-mechanical model}

During gas production or storage, flows are advective in the cleats while it is diffusive in the matrix since micropores are small compared to the gas mean free path. Gas molecules are therefore firstly adsorbed or desorbed at the coal matrix-cleat inter-

face, what establishes a gas concentration gradient which is a driving force for the gas migration in the matrix (Figure 1). 


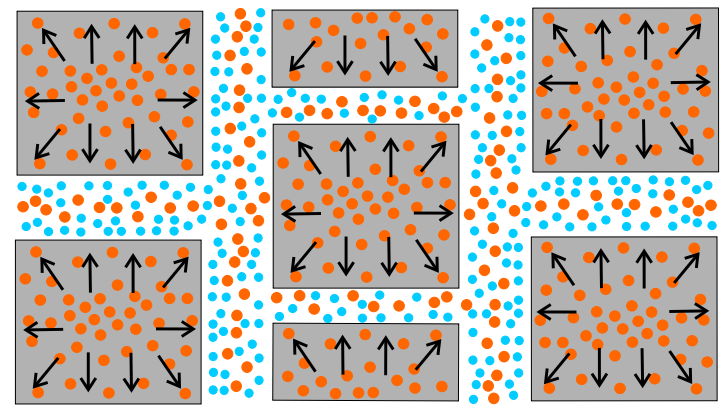

Figure 1: Conceptual hydraulic model.

In the context of CBM or CCS, we are certainly interested in the modelling of this fluid flow problem. However, since it is highly coupled, it can not be treated apart from the mechanical problem. The hydro-mechanical model is presented hereafter first for the matrix and then for the cleats.

\subsection{Matrix}

\subsubsection{Mechanical problem}

The matrix bodies verify the classic mechanical equilibrium Equation (1). Neglecting the gravity term, this equation reads

$$
\frac{\partial \sigma_{i j}}{\partial x_{j}}=0
$$

with $\sigma_{i j}$ the Cauchy stress tensor.

An elastic constitutive law is assumed effective for describing the stress-strain relationship of the coal matrix before failure. The elastic constitutive model relates stress and strain increments through the elastic stiffness tensor $C_{i j k l}$ or inversely with the elastic compliance $D_{i j k l}$ :

$$
\tilde{\sigma}_{i j}=C_{i j k l} \dot{\varepsilon}_{k l} \quad \Leftrightarrow \quad \dot{\varepsilon}_{i j}=D_{i j k l} \tilde{\sigma}_{k l}
$$

where $\dot{\varepsilon}_{i j}$ is the Cauchy strain rate and $\tilde{\sigma}_{i j}$ is the Jaumann stress rate.

Considering an isotropic medium, the elastic constitutive tensors are defined by two independent parameters, e.g. the Young's modulus $E_{m}$ and the Poisson's ratio $v_{m}$. The elastic compliance writes

$$
D_{i j k l}=\frac{1}{E} \cdot\left[\begin{array}{cccccc}
1 & -v & -v & 0 & 0 & 0 \\
-v & 1 & -v & 0 & 0 & 0 \\
-v & -v & 1 & 0 & 0 & 0 \\
0 & 0 & 0 & (1+v) & 0 & 0 \\
0 & 0 & 0 & 0 & (1+v) & 0 \\
0 & 0 & 0 & 0 & 0 & (1+v)
\end{array}\right]
$$


The elastic stiffness tensor $C_{i j k l}$ may be determined by calculating the inverse of the elastic compliance $D_{i j k l}$.

\subsubsection{Hydraulic problem}

Mass balance equation.

Gas is the lonely fluid considered in the matrix. On a unit volume, the mass balance equation of the gas in the matrix is:

$$
\frac{\partial}{\partial t}\left(\rho_{g, A d}\right)+\frac{\partial}{\partial x_{i}}\left(J_{m i}^{g}\right)=0
$$

where the first term is related to the mass variation of the adsorbed gas and the second term is related to the gas diffusion. These two quantities are considered in the following paragraphs.

Density variation.

The classical ideal gas equation of state is used to write the adsorbed gas density as

$$
\rho_{g, A d}=\frac{M_{g}}{R T} p_{g, A d}
$$

where $R$ is the universal gas constant $(8.3143 \mathrm{~J} / \mathrm{mol} \cdot K), T[K]$ is the absolute temperature, $M_{g}$ is the molecular mass of the gas $(0.016 \mathrm{~kg} / \mathrm{mol}$ for methane and $0.044 \mathrm{~kg} / \mathrm{mol}$ for carbon dioxide) and $p_{g, A d}$ is the gas pressure in the matrix, i.e. an adsorbed gas pressure.

\section{Diffusive flows.}

Due to its simplicity, Fick's law (Fick, 1855) is the most popular approach to evaluate diffusive flows. It states the flux in the direction $i$ for the species $g$ diffusing in the matrix $(m)$ is directly proportional to the concentration gradient in that direction:

$$
J_{m i}^{g}=-D_{m}^{g} \frac{\partial C_{g}}{\partial x_{i}}
$$

In this equation, the determination of the phenomenological coefficient $D_{m}^{g}$ is naturally the most critical part of the equation. Rigorously, the thermodynamically correct driving force for diffusion is the chemical potential gradient. Indeed, diffusion is a spontaneous process reducing the total free energy. As the chemical potential generally increases with increasing concentration, it is convenient to express diffusion in term of concentration or mass density. 


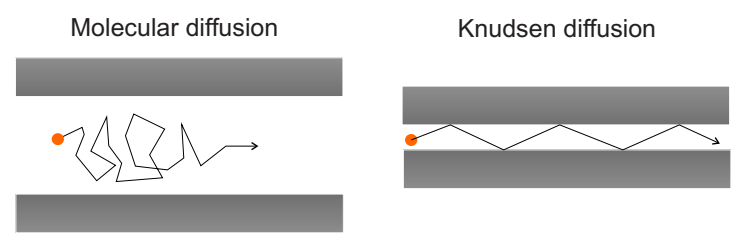

Figure 2: Different types of diffusion.

When the pores are very narrow, diffusing molecules collide with the walls much more frequently than with other molecules. It results in a sliding of the gas molecules along the internal walls of the porous network, it is the Knudsen diffusion (Figure 2). The Knudsen number serves to distinguish which type of diffusion is predominant in the transport process, it is defined as follows (Cussler, 2009):

$$
\kappa_{n}=\frac{l}{d_{p}}
$$

where $l$ is the gas mean free path and $d_{p}$ is a characteristic length such as the mean pore diameter of the porous media. Molecular diffusion (molecule-molecule collisions) prevails when the pore diameter is greater than ten times the mean free path (i.e. $\kappa_{n}$ lower than 0.1). But if the mean free path is greater than ten times the pore diameter $\left(\kappa_{n}\right.$ greater than 10$)$, Knudsen diffusion (molecule-wall collisions) may be assumed. In the intermediate, Knudsen diffusion and molecular diffusion compete with one another. For liquids, the Knudsen number is generally small because the mean free path is a few angstroms. For gas, the mean free path can be estimated as (Cussler, 2009):

$$
l=\frac{4 k_{B} T}{\pi d_{g}^{2} p_{g}}
$$

where $d_{g}$ is the collision diameter of the gas molecule $\left(3.3 \AA\right.$ for $\mathrm{CO}_{2}$ and $3.8 \AA$ for $\mathrm{CH}_{4}$ (Ismail et al., 2015)), $k_{B}$ is the Boltzmann constant, $T[\mathrm{~K}]$ is the temperature and $p_{g}[\mathrm{~Pa}]$ is the pressure of the gas. For any given adsorbent and adsorbate, there is therefore a transition from molecular diffusion at high pressures to Knudsen flow at low pressures. For methane and coal, it has been estimated that the mean free path of the methane molecule at standard conditions is about $50 \mathrm{~nm}$, it is substantially lower in deep coal seams where higher pressure is encountered (Shi and Durucan, 2003). However, gas diffusion in the coal matrix is more controlled by Knudsen diffusion due the extremely small size of the pores of the matrix (lower than $2 \mathrm{~nm}$ ).

The Knudsen diffusion coefficient may be estimated by analogy of the kinetic theory of rigid spheres that predicts (Cussler, 2009):

$$
D_{m K}^{g}=\frac{1}{3} d_{p} v_{g}
$$

where $v_{g}$ is the molecular velocity of the gas. From the kinetic energy theory of 
molecules, we have:

$$
\frac{1}{2} M_{g} v_{g}^{2}=k_{B} T
$$

The Knudsen diffusion coefficient is then:

$$
D_{m K}^{g}=\frac{d_{p}}{3} \sqrt{\frac{2 k_{B} T}{M_{g}}}
$$

Actually, in fine micropores (lower than 1 $\mathrm{nm}$ ), the diffusing molecules never really escape the potential field of the adsorbing surface. Therefore, their transport is an activated process consisting by jumps from one adsorption site to the other (Shi and Durucan, 2003). This transport process is thus more similar to surface diffusion (Figure 3) involving a diffusion coefficient $D_{m S}^{g}\left[\mathrm{~m}^{2} / \mathrm{s}\right]$ which is proportional to the mean-square jump distance $\left[\mathrm{m}^{2}\right]$ and the jump frequency $\left[\mathrm{s}^{-1}\right]$.

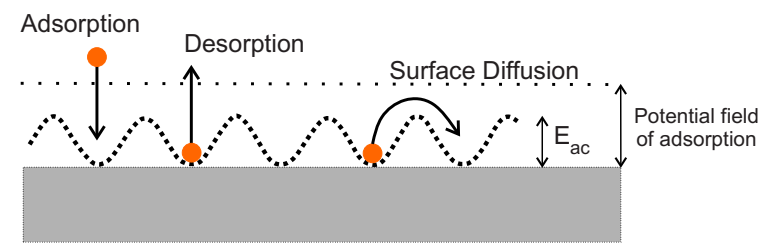

Figure 3: Schematic diagram of surface diffusion. Modified from (Wu et al., 2015).

As different mechanisms may be combined, the diffusion process is a complex physical phenomenon. It is assumed it may be represented by a global phenomenological parameter $D_{m}^{g}\left[m^{2} / s\right]$ to evaluate the diffusive flow $J_{m i}^{g}$ with Equation (6) in which the concentration $C_{g}$ is replaced by the density $\rho_{g, A d}$.

\subsubsection{Hydro-mechanical coupling}

A structural rearrangement of the matrix is induced by the variation of the adsorbed gas pressure (Karacan, 2003). As a consequence, the matrix swells or shrinks. The variation of the volumetric sorption-induced strain $\dot{\varepsilon}_{v_{s}}$ is approximately proportional to the variation of the adsorbed gas content ${ }^{1}$ :

$$
\dot{\varepsilon}_{v_{s}}=\beta_{\varepsilon} \frac{M_{g}}{R T} \frac{1}{\rho_{g, s t d} \rho_{c}} \frac{\partial p_{g, A d}}{\partial t}
$$

where $\beta_{\varepsilon}\left[\mathrm{kg} / \mathrm{m}^{3}\right]$ is the volumetric strain coefficient and $\rho_{g, s t d}$ and $\rho_{c}$ are respectively the gas density at standard conditions and the coal density. Assuming an isotropic swelling/shrinkage of the matrix, the mechanical constitutive equation becomes ${ }^{1}$ :

$$
\tilde{\sigma}_{i j}=C_{i j k l}\left(\dot{\varepsilon}_{k l}-\frac{1}{3} \dot{\varepsilon}_{v_{s}} \delta_{i j}\right)
$$

\footnotetext{
${ }^{1}$ Positive tensile strain and stress.
} 
with $\delta_{i j}$ the Kronecker's symbol.

Note the swelling may, to some extent, deviates from a linear relationship with the gas content. However, given its wider and easier applicability, the linear law is often chosen (Cui and Bustin, 2005). Using a non-linear model would not modify the understanding on the hydro-mechanical behaviour of the material.

\subsection{Cleats}

Considering a matchstick geometry (Seidle et al., 1992), coal is defined by some matrix blocks of width $w$ separated by cleats (Figure 4). The mechanical aperture of the cleats is $h$. However, a hydraulic aperture $h_{b}$ is considered for the hydraulic problem. In $3 \mathrm{D}$, the fracture is defined by the plane $\left(x_{1}, x_{3}\right)$.

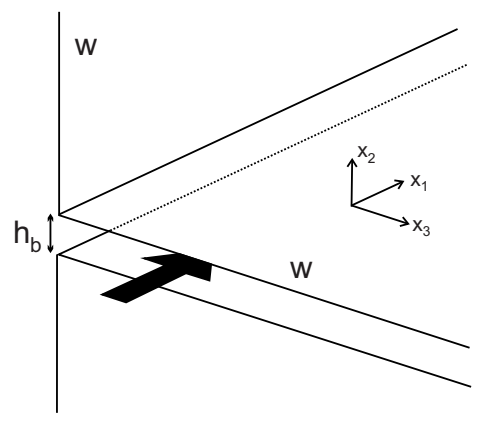

Figure 4: Definition of the geometry of the cleat with a flow along $x_{1}$ Modified from (Reiss, 1980).

\subsubsection{Mechanical problem}

The relation between the normal stress rate $\dot{\sigma}$ and the fracture closure $\dot{h}$ is defined via the normal stiffness $K_{n}{ }^{1}$ :

$$
\dot{\sigma}=K_{n} \cdot \dot{h}
$$

Due to the deforming asperities, this relation should be non-linear (Gens et al., 1990) (Figure 5). A hyperbolic law is used to account for the evolution of the stiffness with the fracture closure (Goodman, 1976; Bandis et al., 1983):

$$
K_{n}=\frac{K_{n}^{0}}{\left(1+\frac{\Delta h}{h_{0}}\right)^{2}}
$$

where $K_{n}^{0}$ is the stiffness corresponding to the aperture $h_{0}$ and $\Delta h=h-h_{0}$. 


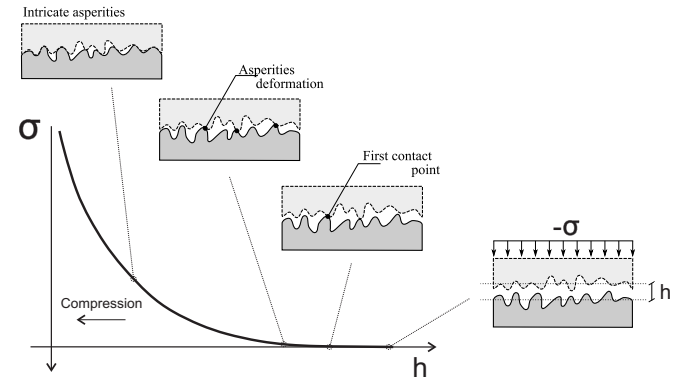

Figure 5: Constitutive law describing the normal behaviour of a rough rock joint. Modified from (Cerfontaine et al., 2015).

For the tangential behaviour of the fracture, two states can be distinguished: the stick and the slip states. In the first state, two points in contact are stuck together. In the second state, there is a relative tangential displacement in the plane of the fracture. The tangential variation of displacement in the direction $i$ (1 and 3$), \dot{s}_{i}$, is related to the shear stress in that direction, $\dot{\tau}_{i}$, as ${ }^{1}$

$$
\dot{\tau}_{i}=K_{s} \cdot \dot{s}_{i}
$$

where $K_{s}$ is the shear stiffness of the fracture.

The two states can be distinguished by a constitutive criterion. One of the most basic is probably the Mohr-Coulomb criterion. Using a single variable $\mu$ representing a friction coefficient, the criterion reads

$$
f=\|\tau\|-\mu \cdot \sigma
$$

where $\|\tau\|$ is the norm of the shear stresses. The criterion is represented in Figure 6.

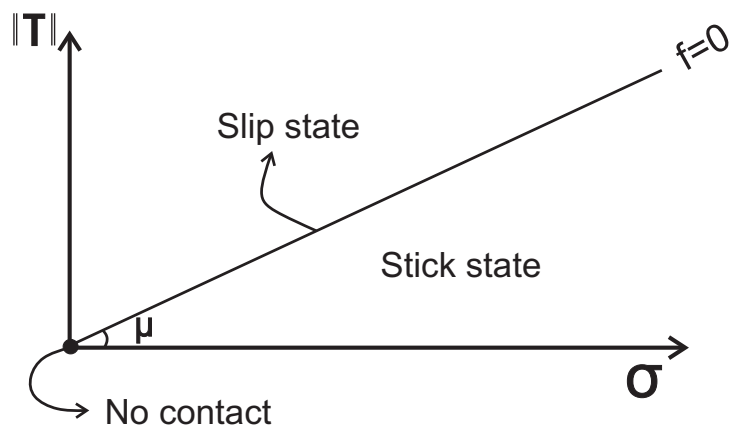

Figure 6: Mohr-Coulomb criterion.

When the combination of tangential and normal stresses lies below the criterion $(f<0)$, it is a tangential stick state. It can be compared to an elastic state. In this case, 
the incremental relation between stresses and displacements reads

$$
\left\{\begin{array}{c}
\dot{\tau}_{1} \\
\dot{\sigma} \\
\dot{\tau}_{3}
\end{array}\right\}=\left[\begin{array}{ccc}
K_{s} & 0 & 0 \\
0 & K_{n} & 0 \\
0 & 0 & K_{s}
\end{array}\right]\left\{\begin{array}{c}
\dot{s}_{1} \\
\dot{h} \\
\dot{s}_{3}
\end{array}\right\}
$$

If the stress state lies on the criterion $(f=0)$, then it is a slip state. Once this state is reached, the elastoplastic constitutive relation is then

$$
\left\{\begin{array}{c}
\dot{\tau}_{1} \\
\dot{\sigma} \\
\dot{\tau}_{3}
\end{array}\right\}=\left[\begin{array}{ccc}
K_{s}\left(1-\frac{\left(\tau_{1}\right)^{2}}{\|\tau\|^{2}}\right) & -\mu K_{n} \frac{\tau_{1}}{\|\tau\|} & -\mu K_{s} \frac{\tau_{1} \tau_{3}}{\|\tau\|^{2}} \\
0 & K_{n} & 0 \\
-\mu K_{s} \frac{\tau_{1} \tau_{3}}{\|\tau\|^{2}} & -\mu K_{n} \frac{\tau 3}{\|\tau\|} & K_{s}\left(1-\frac{\left(\tau_{3}\right)^{2}}{\|\tau\|^{2}}\right)
\end{array}\right]\left\{\begin{array}{c}
\dot{s}_{1} \\
\dot{h} \\
\dot{s}_{3}
\end{array}\right\}
$$

The cohesion $c$ can be used as second parameter to define the general Mohr-Coulomb criterion. Note that many others constitutive laws also exist, taking into more complex behaviours of fractures with damage for instance. But it is not of primary interest in the context of gas flow modelling and hydraulic fracturing is not modelled in this work.

\subsubsection{Hydraulic problem}

Two kinds of flow are distinguished in the cleats, transverse and longitudinal flows (Figure 7). Transverse flows $q_{T}$ represent the gas transfer between the cleats and the matrix while the longitudinal multiphase flows $q_{L}$ occur in between the fracture walls. The longitudinal flow of the gas species consists of the flow of the dry gas and the flow of the dissolved gas in the water phase. For the sake of simplicity, the hydraulic problem described in the following is written considering only a longitudinal flow along $x_{1}$ and not $x_{3}$.

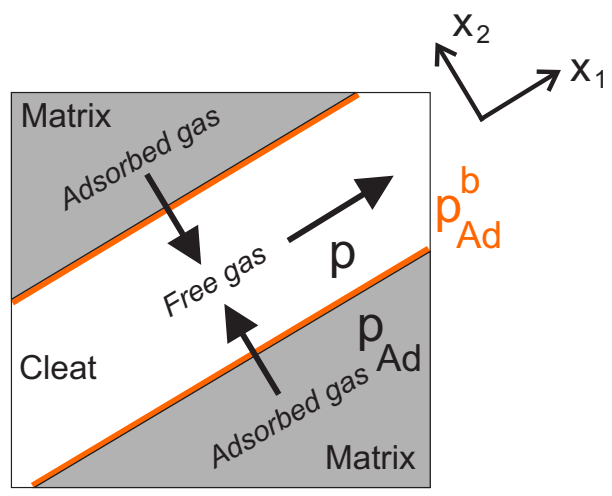

Figure 7: Cleats flow model.

Mass balance equations.

The unsaturated model considers both gas and water in the cleats. The mass balance 
equation for water is:

$$
\frac{\partial}{\partial t}\left(\rho_{w} S_{r} h_{b}\right)+\frac{\partial}{\partial x_{1}}\left(\rho_{w} h_{b} q_{l_{L}}\right)=0
$$

where $\rho_{w}$ is the water density, $S_{r}$ is the degree of saturation, $h_{b}$ is the hydraulic aperture and $q_{l_{L}}$ the liquid advective flows along the fracture. A second longitudinal flow should appear in the equation when 3D conditions are considered. There is no transverse flow since water is not considered in the matrix. Note the cleat is considered as an entirely void volume and no porosity therefore appears in this equation. Moreover, the water vapour is not considered.

Concerning the mass balance equation for gas, it reads:

$$
\begin{aligned}
& \frac{\partial}{\partial t}\left(\rho_{g, f}\left(1-S_{r}\right) h_{b}\right) \\
& \underbrace{+\frac{\partial}{\partial x_{1}}\left(\rho_{g, f} h_{b} q_{g_{L}}\right)+\frac{\partial}{\partial x_{2}}\left(\rho_{g, f} h_{b}\left(q_{g_{T}}^{1}-q_{g_{T}}^{2}\right)\right)}_{\text {Gas phase }} \\
& +\underbrace{\frac{\partial}{\partial t}\left(\rho_{g, f}^{d} S_{r} h_{b}\right)+\frac{\partial}{\partial x_{1}}\left(\rho_{g, f}^{d} h_{b} q_{l_{L}}+S_{r} h_{b} J_{l L}^{g}\right)}_{\text {Dissolved gas in water phase }}=0
\end{aligned}
$$

where $\rho_{g, f}$ and $\rho_{g, f}^{d}$ are respectively the densities of the gas and the dissolved gas in the water. $J_{l L}^{g}$ represents the diffusive flows of the dissolved gas in the liquid phase, it is determined with a Fick's law. $q_{g_{L}}$ represents the longitudinal flow and $q_{g_{T}}^{1}$ and $q_{g_{T}}^{2}$ the two transverse flows. The formulations of these advective flows are described hereafter.

\section{Thermodynamic equilibrium.}

The density of the dissolved gas is obtained with the Henry's law assuming a thermodynamic equilibrium between the dissolved gas and the dry gas (Collin et al., 2002):

$$
\rho_{g, f}^{d}=H_{g} \rho_{g, f}
$$

where $H_{g}$ is the so-called Henry's coefficient $\left(H_{g}=0.0347\right.$ for methane and $H_{g}=$ 0.8317 for carbon dioxide).

Density variations.

The density $\rho_{w}$ of the liquid phase evolves with the pressure $p_{w}$ according to:

$$
\rho_{w}=\rho_{w_{0}}\left(1+\frac{p_{w}-p_{w_{0}}}{\chi_{w}}\right)
$$

where $\rho_{w_{0}}$ is the liquid density at the pressure $p_{w_{0}}$ and $1 / \chi_{w}$ is the liquid compressibility. At $20^{\circ} \mathrm{C}, 1 / \chi_{w}=5 \cdot 10^{-10} \mathrm{~Pa}^{-1}$. 
The classical ideal gas equation of state is used to write the gas density in the fractures as

$$
\rho_{g, f}=\frac{M_{g}}{R T} p_{g, f}
$$

where $p_{g, f}$ is the gas pressure in the fractures.

\section{Gas transfer.}

The quantity of gas stored in the matrix in function of the pressure in the cleats depends on the Langmuir's isotherm. Thence, in order to evaluate the gas transfer between the matrix and the fractures, the fracture pressure is translated with the isotherm into an adsorbed gas pressure. Given a pressure $p$ in the cleat, the gas pressure in the matrix must tend (from its boundary) to:

$$
p_{g, A d}^{b}=\frac{R T}{M_{g}} \cdot \rho_{g, s t d} \cdot \rho_{c} \frac{V_{L} \cdot p}{P_{L}+p}
$$

where the pressure in the cleat can be evaluated by weighting the water and gas pressures by the saturation of each phase:

$$
p=S_{r} p_{w}+\left(1-S_{r}\right) p_{g, f}
$$

Then, the gas transfer from the matrix to the cleat is proportional to the difference between the adsorbed gas pressure at the boundary of the matrix and the limit pressure $p_{g, A d}^{b}$ :

$$
q_{g_{T}}=-T_{t} \cdot \frac{M_{g}}{R T} \cdot\left(p_{g, A d}^{b}-p_{g, A d}\right)
$$

where $T_{t}$ is the gas transverse transmissivity of the fracture. Actually, for unsaturated conditions, Equation (27) can be used without fear for desorption (i.e $p_{g, A d}^{b}$ is lower than $\left.p_{g, A d}\right)$. But for adsorption, it must be verified that gas is available to be adsorbed.

\section{Channel flow model.}

The motion of compressible Newtonian fluids is described by the Navier-Stokes equations. These equations result from the application of the Reynolds transport theorem to the fluid density $\rho$ and the momentum $\rho \mathbf{v}$. Considering a constant density and if there is any source or sink of mass, the conservation of mass reduces to conservation of volume:

$$
\frac{\partial v_{i}}{\partial x_{i}}=0
$$

According to Cauchy momentum equation, the source term in the balance of the momentum may be decomposed into the surface forces (normal and shear stresses) and the body forces, such as gravity. Considering Equation (28), the conservation of momentum gives the following formulation of the Navier-Stokes equations:

$$
\rho\left(\frac{\partial v_{i}}{\partial t}+v_{j} \frac{\partial v_{i}}{\partial x_{j}}\right)=-\frac{\partial p}{\partial x_{i}}+\mu \frac{\partial^{2} v_{i}}{\partial x_{j}^{2}}+f_{i}
$$


where $\rho$ is the fluid density, $v_{i}$ are the components of the fluid velocity, $t$ is the time, $p$ is the pressure, $\mu$ is the dynamic viscosity and $f_{i}$ is an external body force.

Under the assumption the cleats are two closely-space parallel plates separated by an aperture $h_{b}$ (Figure 4), the geometry is known and we can derive the constitutive flow law directly from Navier-Stokes. This channel flow model is straightforward, it allows to directly link the fluid velocity with the fracture aperture.

Assuming laminar flow $\left(v_{j} \frac{\partial v_{i}}{\partial x_{j}}=0\right)$ and steady state conditions $\left(\frac{\partial v_{i}}{\partial t}=0\right)$, the momentum Equation (29) reduces to Equation (30) when no body force is considered.

$$
\frac{\partial^{2} v_{i}}{\partial x_{j}^{2}}=\frac{1}{\mu} \frac{\partial p}{\partial x_{i}}
$$

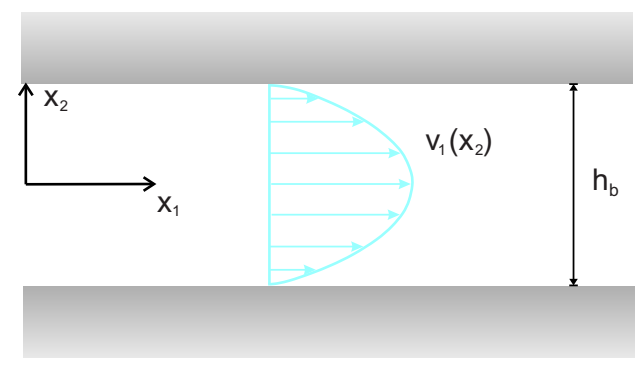

Figure 8: Laminar fluid flow profile between two parallel plates.

The following non-slip boundary conditions are considered at the walls:

$$
v\left(x_{2}= \pm \frac{h}{2}\right)=0
$$

Moreover, the flow is only carried by the $x_{1}$ component: $\frac{\partial p}{\partial x_{2}}=0$. It leads to the following parabolic profile where the maximum velocity is reached in the middle of the channel:

$$
v_{1}\left(x_{2}\right)=-\frac{1}{2 \mu}\left(\left(\frac{h_{b}}{2}\right)^{2}-x_{2}^{2}\right) \frac{\mathrm{d} p}{\mathrm{~d} x_{1}}
$$

Then, averaging the velocity over the thickness $h$ leads to:

$$
q=\left\langle v_{1}\right\rangle=\frac{1}{h_{b}} \int_{-\frac{h_{b}}{2}}^{\frac{h_{b}}{2}} v_{1} d x_{2}=-\frac{h_{b}^{2}}{12} \cdot \frac{1}{\mu} \frac{\mathrm{d} p}{\mathrm{~d} x_{1}}
$$

This channel flow Equation (32) is known as the Hele-Shaw relation (Gustafsson and Vasil'ev, 2006). Using the distance $s$ along the channel to generalize the example, we can write 


$$
q=-\frac{h_{b}^{2}}{12} \cdot \frac{1}{\mu} \frac{\mathrm{d} p}{\mathrm{~d} s}
$$

It is equivalent to the Darcy's equation with a permeability equal to $k=\frac{h_{b}^{2}}{12}$ (Darcy, 1856; Witherspoon et al., 1980), it is function of the fracture aperture.

The channel flow model has been derived with no-slip boundary conditions. However, it may be not the case for gas flow. If the Knudsen number $\left(\kappa_{n}\right)$ is less than 0.01, then viscous flow dominates: the fluid is immobile at the pore wall and Equation (33) can be applied. If $\kappa_{n}$ is comprised between 0.01 and 0.1 , slip flow regime occurs and our flow model should be modified to account this effect. For higher values of $\kappa_{n}$, equations of diffusive flows are employed.

The problem of gas slippage in capillary tubes and its effect on permeability in porous media was first addressed by Klinkenberg (Klinkenberg et al., 1941). A model for the flow between two parallel plates is derived in Appendix A, it gives the permeability should be multiplied by a dimensionless correction factor $f_{c}$ given by:

$$
f_{c}=\left(1+\frac{b}{\bar{p}}\right) \quad \text { with } \quad \frac{b}{\bar{p}}=\frac{6 c \bar{l}}{h_{b}}=6 c \kappa_{n},
$$

where $c \approx 1, \bar{p}$ is the mean gas pressure and $b$ is the Klinkenberg number. For a capillary tube, it has been found that $\frac{b}{\bar{p}}=\frac{4 c \bar{l}}{r}$ with $r$ the effective pore radius (Klinkenberg et al., 1941). Note that the gas permeability is a function of the mean free path $l$ of the gas molecules, it depends therefore on the pressure, the temperature and the nature of the gas. Considering a fracture aperture of few micrometers, the correction factor at reservoir temperature conditions is less than $1 \%$ for a $\mathrm{CO}_{2}$ or $\mathrm{CH}_{4}$ pressure of $1 \mathrm{MPa}$.

Finally, if gas and water flows are simultaneously considered in the cleats, it is necessary to extend the model to unsaturated conditions. In this purpose, relative permeability $k_{r}$ may be introduced as a measure of the reduction in permeability to a given phase that occurs between partially and fully saturated conditions. Some specific relative permeability curves for the fractures are derived in Appendix B. It reads

$$
k_{r w}=\frac{S_{r}^{2}}{2}\left(3-S_{r}\right) \quad ; \quad k_{r g}=\left(1-S_{r}\right)^{3}
$$

The saturation degree is obtained from the retention curve, like the Brooks-Corey equation (see Equation B.33 in Appendix B)

\subsubsection{Hydro-mechanical couplings}

The first coupling is the direct dependency of the permeability on the fracture aperture, which is stress-dependent. To take into account the non-smoothness of the fracture walls, an hydraulic aperture is considered rather than a mechanical one. The hydraulic aperture $h_{b}$ is derived from the mechanical one $h$ according to the following linear 
relation:

$$
h_{b}=h^{\min }+h
$$

It means a fracture which is mechanically closed $(h=0)$ still allows a flow to circulate between the asperities if a non-zero minimal hydraulic aperture $h^{\min }$ is defined (Figure 9). Actually, an exponential law could also be applied to relate the hydraulic aperture to the mechanical aperture (and the JRC) (Barton et al., 1985; Olsson and Barton, 2001).

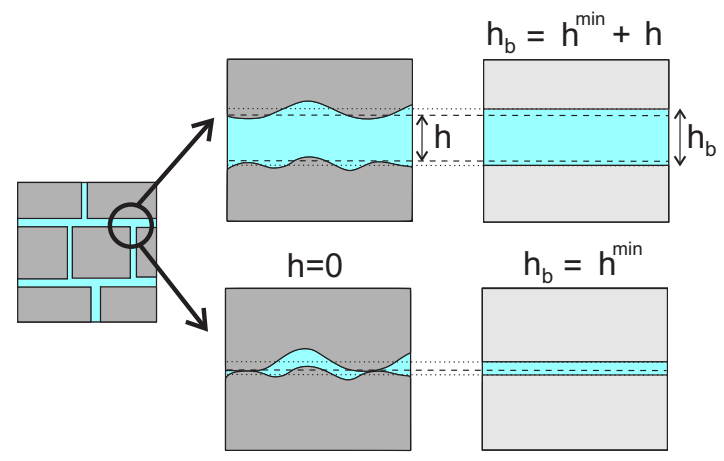

Figure 9: Relation between the hydraulic and the mechanical aperture. Modified from (Marinelli et al., 2016).

A second coupling is created by the storage component. Indeed, the variations of the total masses of water $\dot{M}_{w}$ and gas stored $\dot{M}_{g}$ in the cleat come from a rheological part, i.e the variations of the fluid densities and the saturation of each phase, but also from a geometric part, i.e the opening/closure of the gap and the variation of the surface of the discontinuity:

$$
\dot{M}_{w}=\dot{\rho}_{w} S_{r} h_{b} \Gamma+\rho_{w} \dot{S}_{r} h_{b} \Gamma+\underbrace{\rho_{w} S_{r} \dot{h}_{b} \Gamma+\rho_{w} S_{r} h_{b} \dot{\Gamma}}_{\text {Hydro-mechanical coupling }}
$$

and

$$
\begin{aligned}
\dot{M}_{g} & =\left(\dot{\rho}_{g, f}\left(1-S_{r}\right)+\dot{\rho}_{g, f}^{d} S_{r}+\rho_{g, f}\left(1-\dot{S}_{r}\right)+\rho_{g, f}^{d} \dot{S}_{r}\right) h_{b} \Gamma \\
& +\underbrace{\left(\rho_{g, f}\left(1-S_{r}\right)+\rho_{g, f}^{d} S_{r}\right)\left(\dot{h}_{b} \Gamma+h_{b} \dot{\Gamma}\right)}_{\text {Hydro-mechanical coupling }}
\end{aligned}
$$

where $\Gamma$ is the surface of the discontinuity.

Inversely, the fluid flow within the cleat also influences its mechanical behaviour. The total pressure $\sigma$ acting on each side of the fracture can be decomposed into an effective mechanical pressure $\sigma^{\prime}$ and a fluid pressure $p$ in the fracture defined by Equation (26). Then, the mechanical constitutive law defined in section 2.2.1 must be treated with reference to the effective stress ${ }^{1}$ :

$$
\sigma^{\prime}=\sigma+p
$$

rather than the total pressure $\sigma$. 


\subsection{Continuum formulation}

\subsubsection{Mechanical problem}

For the mechanical problem, let consider two media $\Omega^{1}$ and $\Omega^{2}$ representing for instance two matrix blocks (Figure 10). Their boundaries are termed $\Gamma^{1}$ and $\Gamma^{2}$ with $\Gamma_{c}^{1}$ and $\Gamma_{c}^{2}$ denoting parts of the boundaries where contact is likely to happen.

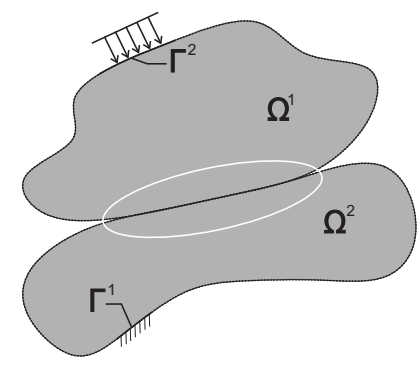

Figure 10: Definition of the mechanical problem.

Each of these solids verifies the mechanical equilibrium Equation (1). Solving the mechanical problem consists in finding the field of displacement verifying the equilibrium under the constraint of a non-zero pressure along the contact zone $\Gamma_{c}$. In this purpose, the weak formulation is obtained considering an admissible virtual velocity field $v_{i}^{*}$. The virtual power principle reads

$$
\sum_{k=1}^{2}\left[\int_{\Omega^{k}} \sigma_{i j} \frac{\partial v_{i}^{*}}{\partial x_{j}} d \Omega\right]=\sum_{k=1}^{2}\left[\int_{\Gamma_{\bar{t}_{i}}^{k}} \bar{t}_{i} v_{i}^{*} d \Gamma+\int_{\Gamma_{c}^{k}} T_{i}^{k} v_{i}^{*} d \Gamma\right]
$$

where $\bar{t}_{i}$ are the imposed tractions and $T_{i}^{k}$ is the projection of the local stress tensor in global coordinates.

\subsubsection{Hydraulic problem}

The hydraulic problem considers the presence of the third volume $\Omega^{3}$ to represent the discontinuity creating a preferential pathway for the fluid flow (Figure 11). This volume $\Omega^{3}$ is bounded by the boundaries $\Gamma_{\tilde{q}}^{1}$ and $\Gamma_{\tilde{q}}^{2}$ corresponding to the regions where $\Omega^{1}$ and $\Omega^{2}$ are close from each other. The fluid flows between $\Omega^{3}$ and the solid bodies $\Omega^{1}$ and $\Omega^{2}$ are non-classical boundary conditions for these bodies since neither the flow nor the pressure is imposed. Elsewhere, imposed flux boundaries on $\Omega^{1}$ and $\Omega^{2}$ are denoted by $I_{\bar{q}}^{k}$ with $k=1,2$. 


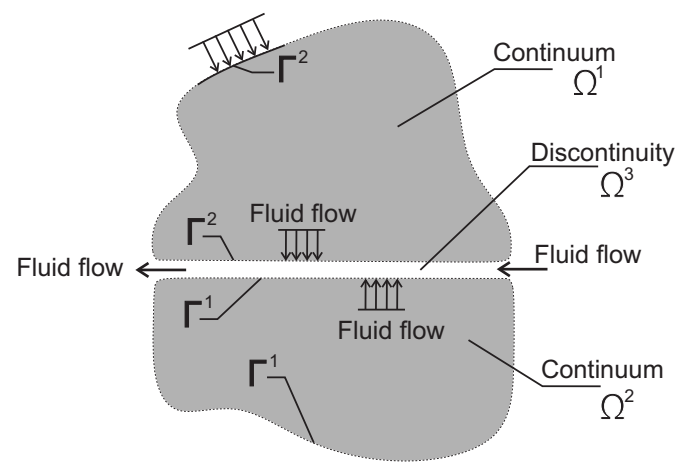

Figure 11: Definition of the flow problem.

For gas, these three volumes verify the hydraulic equilibrium Equations (4) and (21) with a non-classical boundary given by Equation (27). Considering a field of admissible virtual gas pressure $p_{g}^{*}$, the weak form of the virtual power principle is

$$
\begin{aligned}
& \sum_{k=1}^{3}\left[\int_{\Omega^{k}} \dot{S}_{g} p_{g}^{*}-f_{g_{i}}^{k} \frac{\partial p_{g}^{*}}{\partial x_{i}} d \Omega\right]= \\
& \quad \sum_{k=1}^{3}-\left[\int_{\Gamma_{\bar{q}_{g}}^{k}} \bar{q}_{g} p_{g}^{*} d \Gamma+\int_{\Gamma_{\tilde{q}_{g}}^{k}} \tilde{q}_{g} p_{g}^{*} d \Gamma\right]
\end{aligned}
$$

where $k=1,2$ corresponds to the matrix blocks in contact and $k=3$ to the volume of the cleat. For $\Omega^{1}$ and $\Omega^{2}$, the storage term $\dot{S}_{g}$ is the variation of the adsorbed gas mass. For $\Omega^{3}$, it is the mass variation given by Equation (38) over a unit surface of discontinuity. $f_{g_{i}}^{k}$ is the gas flux, advective or diffusive, inside $\Omega^{k} . \bar{q}_{g}$ and $\tilde{q}_{g}$ are the fluid flows along the different boundaries.

The integral over $\Omega^{3}$ may be transformed into a surface integral over $\Gamma_{\tilde{q}_{g}}^{1}$ assuming the inner pressure is constant over the fracture aperture. The flux $f_{g_{i}}^{k}$ is replaced by the longitudinal flux $f_{g_{L}}$ and the flux $\tilde{q}_{g}$ is replaced by the transverse fluxes $f_{g_{T}}^{k}$. The integral over $\Omega^{3}$ reads

$$
\int_{\Gamma_{\tilde{q}_{g}}^{1}}\left[\dot{S}_{g} p_{g}^{*}-f_{g_{L}} \frac{\partial p_{g}^{*}}{\partial x_{1}}\right] h_{b} d \Gamma=-\int_{\Gamma_{\tilde{q}_{g}}^{1}}\left(f_{g_{T}}^{1}-f_{g_{T}}^{2}\right) p_{g}^{*} d \Gamma
$$

where the internal longitudinal flux of the gas species $f_{g_{L}}$ is given by (along $x_{1}$ ):

$$
f_{g_{L}}=\rho_{g, f} q_{g_{L}}+\rho_{g, f}^{d} q_{L_{L}}+S_{r} J_{l_{L}}^{g}
$$

and the two transverse fluxes $f_{g}^{1}$ and $f_{g_{T}}^{2}$ are (along $x_{2}$ ):

$$
f_{g_{T}}^{k}=\rho_{g, f} q_{g_{T}}^{k}
$$

Similarly for water, equilibrium Equation (20) has to be verified. Considering a 
field of admissible virtual water pressure $p_{w}^{*}$, and as water transfers are not allowed between the cleat and the matrix, we finally have

$$
\int_{\Gamma_{\tilde{q}_{w}}^{1}}\left[\dot{S}_{w} p_{w}^{*}-f_{w_{L}} \frac{\partial p_{w}^{*}}{\partial x_{1}}\right] h_{b} d \Gamma=0
$$

where the storage term $\dot{S}_{w}$ is the mass variation $\dot{M}_{w}$ over a unit surface of discontinuity and $f_{w_{L}}=\rho_{w} q_{l_{L}}$.

The final step is the discretization of these governing equations with finite elements.

\section{Finite element formulation}

The hydro-mechanical model presented in the previous section is implemented in the FE code Lagamine. The finite element method is extensively described in the literature (see for instance (Zienkiewicz and Taylor, 2000)). It is certainly the case for the continuum elements used to discretize the matrix. Cleats are discretized with elements of zero-thickness which are probably less usual. A particular attention is therefore given to the interface elements. The elements which served as a basis for the cleat discretization are described for water saturated conditions in (Cerfontaine et al., 2015). This section is mainly focused on the specificities of the formulation of the new model.

\subsection{Space and pressure discretization}

The governing equations are discretized based on a segment-to-segment approach. A three-node formulation is adopted to described the fluid flow through and within the interface. In 3D, it means twelve nodes are required for the complete representation

of the interface (Figure 12). There are four nodes with three mechanical degrees of freedom (coordinates $x, y, z$ ) and at least one fluid degree of freedom per node. For the two sides of the interface, this fluid degree of freedom is the adsorbed gas pressure $p_{g, A d}$ while it is the gas pressure $p_{g, f}$ for the inner nodes.

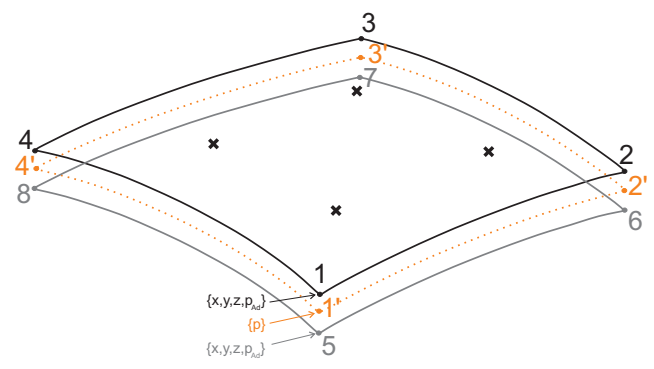

Figure 12: A 3D interface element defined by 12 nodes.

For the matrix, 8-nodes 3D elements are used to match the 3D interface element presented above. The mechanical and hydraulic degrees of freedom are gathered into a vector $u_{m_{n}}$ of generalised coordinates with $m$ the number of degrees of freedom of the 
node $n$. These coordinates are continuously interpolated over the element with classical linear interpolation functions $N^{(n)}$.

\subsection{Energetically equivalent nodal forces}

\subsubsection{External forces}

The energetically equivalent nodal associated to the node $n$ are computed numerically for a single interface element from Equation (40) using a Gauss-scheme. The mechanical nodal forces acting on the boundary of one of the elements are computed as

$$
F_{E, m_{n}}^{\Omega_{e}}=\sum_{I P}\left[t_{m} N^{(n)} \operatorname{det}\left(J_{i j}\right) W_{G}\right]_{I P}
$$

with $m=1,2,3$ the mechanical degrees of freedom and $n=1,2,3,4$ or $n=5,6,7,8$ the nodes used to compute the mechanical forces on the first side of the interface $\Gamma_{c}^{1}$ or the other side $\Gamma_{c}^{2}$. In both cases, the imposed traction $t_{m}$ and the components of the Jacobian matrix $J_{i j}$ of the transformation to the isoparametric system are computed with respect to the local system of coordinates of $\Gamma_{c}^{1}$. $W_{G}$ is the Gauss weight corresponding to the integration point $I P$.

For the flow problem, transverse flows involve energetically equivalent external forces related to gas degrees of freedom. For water, it is not considered but for gas, the contribution of the interface to the external virtual power is derived from Equation (42). It is numerically computed on the boundary of $\Omega^{1}$ according to

$$
F_{E, 4_{n}}^{\Omega_{e}}=\sum_{I P}\left[\left(f_{g_{T}}^{1}-f_{g_{T}}^{2}\right) N^{(n)} \operatorname{det}\left(J_{i j}\right) W_{G}\right]_{I P}
$$

with $f_{g_{T}}^{1}$ and $f_{g_{T}}^{2}$ defined by Equation (44) instead of the classical definition based on a difference between the interface inner node and the solid node.

\subsubsection{Internal forces}

The energetically equivalent internal forces due to the gas flows along the interface are derived from Equation (42) adapted in 3D. It is numerically computed according to

$$
F_{I, 4_{n}}^{\Omega_{e}}=\sum_{I P}\left[\left(\dot{S}_{g} N^{(n)}-f_{g_{L} 1} \frac{\partial N^{(n)}}{\partial x_{1}}-f_{g_{L 2}} \frac{\partial N^{(n)}}{\partial x_{2}}\right) \operatorname{det}\left(J_{i j}\right) W_{G}\right]_{I P}
$$

Similarly for water, the energetically equivalent internal forces due to the longitudinal water flows inside the interface $f_{w_{L}}$ is derived from Equation (45).

\subsubsection{Out of balance forces}

Internal and external nodal forces are gathered together to compute the global vectors $F_{I, m_{n}}$ and $F_{E, m_{n}}$. Solving the numerical problem consists to obtain the equality between these two vectors. The out of balance forces vector is computed as

$$
F_{O B, m_{n}}=F_{E, m_{n}}-F_{I, m_{n}}
$$


In order to obtain $F_{O B, m_{n}}=0$, generalised coordinates $\Delta u_{m_{n}}$ are iteratively corrected by:

$$
\Delta u_{m_{n}}=-\left[\frac{\partial F_{O B, l_{k}}^{t}}{\partial u_{m_{n}}}\right]^{-1} F_{O B, l_{k}}^{t}=-\left[K_{l_{k} m_{n}}^{t}\right]^{-1} F_{O B, l_{k}}^{t}
$$

where $K_{l_{k} m_{n}}^{t}$ is the stiffness matrix at time step $t$.

Note the fluid flow problem is naturally time-dependent and requires the discretization of time. It is assumed the media in contact are initially in equilibrium at a given time $t\left(F_{O B, m_{n}}=0\right)$ and the equilibrium of the discretized system is then written at $t+\Delta t$ with an implicit scheme.

\subsection{Stiffness matrix}

The stiffness matrix $K_{l_{k} m_{n}}$ is computed analytically by derivation of out of balance forces:

$$
K_{l_{k} m_{n}}=\frac{\partial F_{O B, l_{k}}}{\partial u_{m_{n}}}
$$

A description of the components of the stiffness matrix for the interface elements may be found in (Cerfontaine et al., 2015), it was adapted to gas degrees of freedom by taking into account the gas equation of state (24).

Finally, for the continuum elements discretizing the matrix, the shrinkage/swelling due to the adsorbed gas pressure evolution is taken into account like it would be done for thermal dilatation in the code. Thence, from the constitutive Equation (13), the couplings terms of the stiffness matrix accounting for the influence of the adsorbed gas on the mechanics read

$$
\frac{\partial_{F_{O B, i_{k}}}}{\partial_{p_{g, A d_{n}}}}=\sum_{I P}\left[\frac{1}{3} \beta_{\varepsilon} \frac{M_{g}}{R T} \frac{1}{\rho_{g, s t d} \rho_{c}} \frac{(1-v) E}{(1+v)(1-2 v)} \frac{\partial_{N}(k)}{\partial_{x_{i}}} \operatorname{det}\left(J_{i j}\right) W_{G}\right]
$$

with $i=1,2,3$ the three mechanical degrees of freedom.

\section{Analytical validation}

In order to validate the implementation of the model in the finite element code, basic simulations are completed to compare the numerical results to analytical solutions. Actually, many different analytical models were developed in the literature to take into account the hydro-mechanical couplings influencing the permeability evolution (Pan and Connell, 2012). Most of these models are based on the cleat porosity evolution. The typical model found in the literature is an exponential equation. 


\subsection{Exponential model}

From Equation (33), the permeability governing the fracture flow in Darcy's equation is ${ }^{2}$

$$
k_{\text {cleat }}=\frac{h^{2}}{12}
$$

Considering a fractured rock with a spacing $w$ between a set of parallel fractures, the equivalent permeability of this set is

$$
k=k_{\text {cleat }} \cdot \frac{h}{w}=\frac{h^{3}}{12 w}
$$

From this cubic law, the permeability evolution may be approximated as (Seidle et al., 1992):

$$
k=k_{0}\left(\frac{\phi}{\phi_{0}}\right)^{3}
$$

where $k$ is the permeability and $\phi$ the fracture porosity. The index 0 denotes a reference state.

Furthermore, the porosity evolution is generally expressed in terms of pore and bulk strains, respectively $\varepsilon_{p}$ and $\varepsilon_{b}$ (Cui and Bustin, 2005):

$$
\frac{\mathrm{d} \phi}{\phi_{0}}=\mathrm{d} \varepsilon_{b}-\mathrm{d} \varepsilon_{p}
$$

These strains increments may be decomposed into mechanistic and swelling strains increments. Then, the mechanistic strains increments are expressed in terms of stiffness or compressibility and pressure increments (confining and pore pressures) (Zimmerman et al., 1986). Neglecting the cleat stiffness in comparison to the bulk stiffness and, under the approximation of constant cleat stiffness, Equations (55) and (56) finally give (rock mechanics convention) (Cui and Bustin, 2005; Connell et al., 2010):

$$
\begin{gathered}
k=k_{0} \cdot \exp \left[-3\left(\frac{1}{K_{p}}\left(\left(\sigma-\sigma_{0}\right)-\left(p-p_{0}\right)\right)\right.\right. \\
\left.\left.+\left(\varepsilon_{p_{s}}-\varepsilon_{p_{s 0}}\right)-\left(\varepsilon_{b_{s}}-\varepsilon_{b_{s 0}}\right)\right)\right]
\end{gathered}
$$

where $k_{0}$ is the reference permeability for the reference stress $\sigma_{0}$ and the reference pore pressure $p_{0}$, and $K_{p}$ is the cleat stiffness ${ }^{3}$. The stress $\sigma$ includes the boundary conditions and the model may be expanded for different boundaries. The subscript $s$ denotes swelling quantities. In practice, the pore swelling strain $\varepsilon_{p_{s}}$ is difficult to measure and it is sometimes assumed it is equal to the bulk swelling strain $\varepsilon_{b s}$ (Cui and Bustin, 2005). For instance, for reservoir boundary conditions, this assumption leads to the model developed by Shi and Durucan (Shi and Durucan, 2004; Cui and Bustin,

\footnotetext{
${ }^{2}$ For the sake of simplicity, the hydraulic aperture was assimilated to the mechanical aperture $h$ in these developments.

${ }^{3}$ The dimension of this stiffness differs from the stiffness used in Equation (14), there are related by $K_{p}=h \cdot K_{n}$.
} 
2005):

$$
k=k_{0} \cdot \exp \left[\frac{3}{K_{p}}\left(\frac{v_{m}}{1-v_{m}}\left(p-p_{0}\right)-\frac{E_{m}}{3\left(1-v_{m}\right)}\left(\varepsilon_{v_{s}}-\varepsilon_{v_{s_{0}}}\right)\right)\right]
$$

However, Connell et al (Connell et al., 2010) suggested the magnitude of $\varepsilon_{p_{s}}$ is higher than $\varepsilon_{b s}$ and related the two quantities through a fitting parameter $\gamma$ (Connell et al., 2010):

$$
\varepsilon_{p_{s}}=\gamma \cdot \varepsilon_{b_{s}}
$$

More recently, some authors linked the relationship between $\varepsilon_{p_{s}}$ and $\varepsilon_{b_{s}}$ to the fracture porosity (Lu et al., 2016; Peng et al., 2017), but it still requires some fitting parameters. In the following, the analytical solution is determined based on the equilibrium between the matrix block and the fracture instead of the bulk volume.

\subsection{Analytical solution}

Due to the evolution of the pressure in the fracture or the swelling of the matrix, the fracture aperture is not constant:

$$
h=h_{0}+\Delta h
$$

where the subscript 0 denotes the initial configuration and $\Delta$ the increment. So, from Equation (54), the permeability evolution is given by:

$$
k=\frac{\left(h_{0}+\Delta h\right)^{3}}{12 w}
$$

The fracture aperture is stress-dependent and it is determined stating a stress equilibrium between the fracture and the matrix.

For the fracture, the evolution of the normal effective stress (rock mechanics convention) reads

$$
\left(\sigma-p_{g, f}\right)=\left(\sigma_{0}-p_{g, f_{0}}\right)+\Delta\left(\sigma-p_{g, f}\right)
$$

where $\sigma$ is the normal total stress and $p_{g, f}$ is the gas pressure in the fracture. As stated by Equation (14), the variation of the normal effective stress is related to the variation of the fracture aperture through

$$
\mathrm{d}\left(\sigma-p_{g, f}\right)=-K_{n} \cdot \mathrm{d} h
$$

where $K_{n}$ is the normal stiffness of the fracture

For the matrix, the stress evolution reads

$$
\sigma_{i j}=\sigma_{i j_{0}}+\Delta \sigma_{i j}
$$

where $\Delta \sigma_{i j}$ is given by the Hooke's mechanical law taking into account the swelling behaviour:

$$
\Delta \sigma_{i j}=2 G_{m} \Delta \varepsilon_{i j}+\lambda_{m} \Delta \bar{\varepsilon} \delta_{i j}+K_{m} \Delta \varepsilon_{v_{s}} \delta_{i j}
$$

where $\varepsilon_{i j}$ is the strain tensor, $\bar{\varepsilon}=\varepsilon_{i j} \delta_{i j}$ with $\delta_{i j}$ the Kronecker's symbol, $\Delta \varepsilon_{v_{s}}$ is the volumetric sorption-induced strain, $G_{m}$ is the Coulomb's modulus, $\lambda_{m}$ is the first Lamé 
parameter and $K_{m}$ is the bulk modulus of the matrix. All these elastic moduli may also be replaced by $E_{m}$ and $v_{m}$, respectively the Young's modulus and the Poisson's ratio:

$$
\begin{gathered}
G_{m}=\frac{E_{m}}{2\left(1+v_{m}\right)} \\
\lambda_{m}=\frac{E_{m} v_{m}}{\left(1+v_{m}\right)\left(1-2 v_{m}\right)} \\
K_{m}=\frac{E_{m}}{3\left(1-2 v_{m}\right)}
\end{gathered}
$$

Thence, Equation (65) can also be written as

$$
\begin{aligned}
\Delta \sigma_{i j}= & \frac{E_{m}}{1+v_{m}} \Delta \varepsilon_{i j}+\frac{E_{m} v_{m}}{\left(1+v_{m}\right)\left(1-2 v_{m}\right)} \Delta \bar{\varepsilon} \delta_{i j} \\
& +\frac{E_{m}}{3\left(1-2 v_{m}\right)} \Delta \varepsilon_{v_{s}} \delta_{i j}
\end{aligned}
$$

At equilibrium between the fracture and the matrix pressure, the sorption-induced strain $\Delta \varepsilon_{v_{s}}$ may be expressed in term of pressure in the fracture. For instance, it can be linearly linked to the Langmuir's isotherm:

$$
\Delta \varepsilon_{v_{s}}=\beta_{\varepsilon}\left(\frac{V_{L} \cdot p_{g, f}}{P_{L}+p_{g, f}}-\frac{V_{L} \cdot p_{g, f_{0}}}{P_{L}+p_{g, f_{0}}}\right)
$$

where $\beta_{\varepsilon}$ is a linear coefficient, and $V_{L}$ and $P_{L}$ are the Langmuir's parameters. The analytical solution is not restricted to any particular relationship between the sorptioninduced strain. A linear relationship is chosen like it was implemented in the finite element code.

\subsubsection{Constant fracture stiffness}

First, if a constant normal stiffness is considered for the fracture, then there is a linear relationship between the fracture closure and the normal stress given by Equation (63). Thence, Equation (62) gives

$$
\sigma=\sigma_{0}+\Delta p_{g, f}-K_{n} \cdot \Delta h
$$




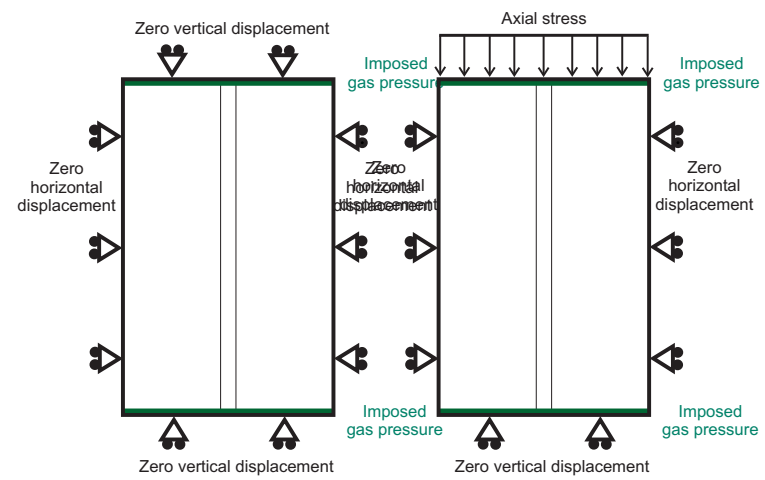

(a) Full rigid boundaries.

(b) Reservoir conditions.

Figure 13: One vertical fracture delimited by two half blocks and boundary conditions considered.

Considering a vertical fracture delimited by two half matrix blocks, from Equations (64) and (71), the horizontal equilibrium reads

$$
\Delta p_{g, f}-K_{n} \cdot \Delta h=\Delta \sigma_{x x}
$$

Finally, $\Delta h$ is found by solving Equation (72) where $\Delta \sigma_{x x}$ depends on $\Delta h$ through the boundary conditions introduced in Equation (69). Two types are considered, full rigid boundaries and in situ reservoir conditions.

\section{Full rigid boundaries.}

With full rigid boundary conditions, the strains in the matrix are $\Delta \varepsilon_{x x}=\frac{\Delta h}{w_{0}}$ and $\Delta \varepsilon_{y y}=\Delta \varepsilon_{z z}=0$. Introducing these conditions in Equation (69) directly gives

$$
\begin{aligned}
\Delta \sigma_{x x} & =\frac{E_{m}\left(1-v_{m}\right)}{\left(1+v_{m}\right)\left(1-2 v_{m}\right)} \frac{\Delta h}{w}+\frac{E_{m}}{3\left(1-2 v_{m}\right)} \Delta \varepsilon_{v_{s}} \\
& =M_{m} \frac{\Delta h}{w_{0}}+K_{m} \Delta \varepsilon_{v_{s}}
\end{aligned}
$$

where $M_{m}=\frac{E_{m}\left(1-v_{m}\right)}{\left(1+v_{m}\right)\left(1-2 v_{m}\right)}$ is the P-wave modulus of the matrix. Introducing this stress increment into Equation (72), the closure is finally given by

$$
\Delta h=w_{0} \cdot \frac{\Delta p_{g, f}-K_{m} \Delta \varepsilon_{v_{s}}}{K_{n} \cdot w_{0}+M_{m}}
$$

The model for the permeability evolution is finally obtained introducing this equation into Equation (61).

Reservoir boundaries.

In situ, rigid boundaries should be considered only horizontally: $\Delta \varepsilon_{x x}=\frac{\Delta h}{w_{0}}$ and 
$\Delta \varepsilon_{y y}=0$, while vertically we have $\Delta \sigma_{z z}=0$. From these boundary conditions, we can write

$$
\left\{\begin{array}{l}
\frac{1}{E_{m}} \Delta \sigma_{x x}-\frac{v_{m}}{E_{m}} \Delta \sigma_{y y}+\frac{1}{3} \Delta \varepsilon_{v_{s}}=\frac{\Delta h}{w_{0}} \\
\frac{1}{E_{m}} \Delta \sigma_{y y}-\frac{v_{m}}{E_{m}} \Delta \sigma_{x x}+\frac{1}{3} \Delta \varepsilon_{v_{s}}=0
\end{array}\right.
$$

Then, by solving this system of equations, we find the following stress increment:

$$
\Delta \sigma_{x x}=\frac{E_{m}}{\left(1-v_{m}^{2}\right)} \frac{\Delta h}{w_{0}}+\frac{E_{m}}{3\left(1-v_{m}\right)} \Delta \varepsilon_{v_{s}}
$$

Hence, introducing this stress increment in Equation (72), the closure is given by

$$
\Delta h=w_{0} \cdot \frac{\Delta p_{g, f}-\frac{E_{m}}{3\left(1-v_{m}\right)} \Delta \varepsilon_{v_{s}}}{K_{n} \cdot w_{0}+\frac{E_{m}}{\left(1-v_{m}^{2}\right)}}
$$

\subsubsection{Hyperbolic fracture stiffness}

The evolution of the normal stiffness with the fracture closure given by Equation (15) is now considered. Then, integrating Equation (63) gives this time

$$
\Delta\left(\sigma-p_{g, f}\right)=-\int_{h_{0}}^{h} \frac{K_{n}^{0}}{\left(1+\frac{h-h_{0}}{h_{0}}\right)^{2}} \mathrm{~d} h=-\frac{K_{n}^{0} h_{0}}{h} \cdot \Delta h
$$

Then, the following equation has to be solved:

$$
\Delta p_{g, f}-\frac{K_{n}^{0} h_{0}}{h_{0}+\Delta h} \cdot \Delta h=\Delta \sigma_{x x}
$$

Considering full rigid boundaries, Equation (74) is introduced in Equation (80) and the second order equation to solve to find $\Delta h$ is

$$
\Delta p_{g, f}-\frac{K_{n}^{0} h_{0}}{h_{0}+\Delta h} \cdot \Delta h=M_{m} \frac{\Delta h}{w_{0}}+K_{m} \Delta \varepsilon_{v_{s}}
$$

Considering reservoir boundaries conditions as described above, Equation (77) is introduced in Equation (80), it gives

$$
\Delta p_{g, f}-\frac{K_{n}^{0} h_{0}}{h_{0}+\Delta h} \cdot \Delta h=\frac{E_{m}}{\left(1-v_{m}^{2}\right)} \frac{\Delta h}{w_{0}}+\frac{E_{m}}{3\left(1-v_{m}\right)} \Delta \varepsilon_{v_{s}}
$$

The permeability models for the two types of boundaries are obtained introducing the solutions of these second degree equations into Equation (61) and keeping the positive permeability. It is plotted in the following section for a given set of parameters. 


\subsection{Analysis and validation}

Figure 14 and 15, considering respectively a constant fracture stiffness and a hyperbolic variation of the stiffness, present the analytical evolutions obtained for the two different boundary conditions. The decrease of the permeability is logically more important with the full rigid boundaries than when the displacement is allowed vertically. The parameters are given in Table 1, the sorption and mechanical parameters of the matrix come from an experimental study carried out in laboratory.

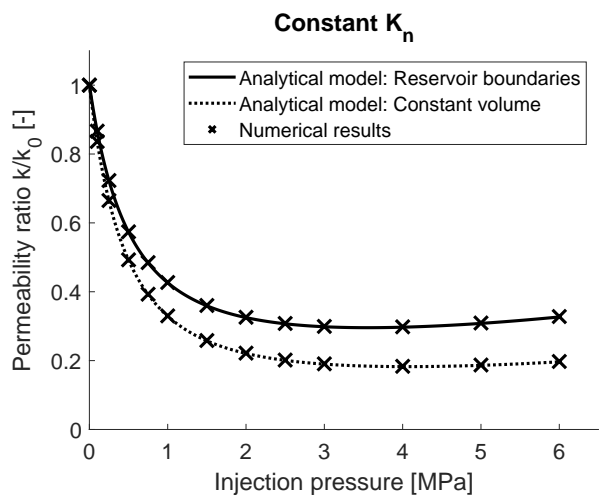

Figure 14: Constant fracture stiffness: Numerical results versus analytical model.

The numerical results are compared to the analytical solutions to validate the implementation of our model. Each numerical result represents the stabilized permeability after the diffusion of the gas in the matrix from the increase of the pressure in the fracture, what induces the swelling gradually. This transient aspect is not taken into account in the analytical model. It would require to use some shape factors (Barenblatt et al., 1960; Warren et al., 1963; Bertrand et al., 2017) in the analytical solution.

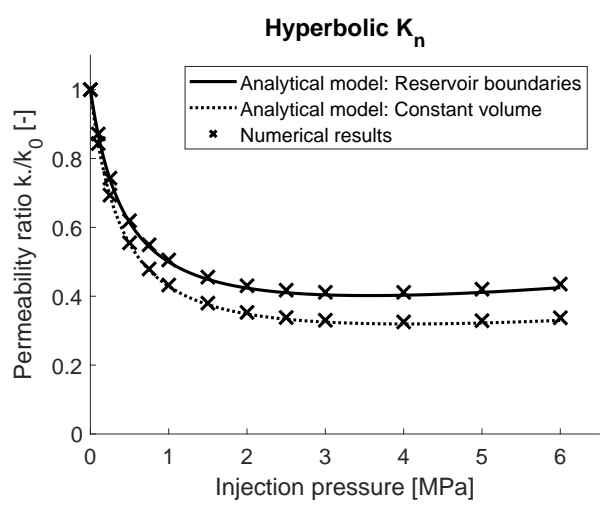

Figure 15: Hyperbolic fracture stiffness: Numerical results versus analytical model.

For the hyperbolic evolution of the fracture stiffness in Figure 15, the initial fracture aperture and stiffness were taken the same as the constant case. As the stiffness 
increases with the closure of the fracture, the permeability is less decreased compared to the results obtained with the constant stiffness behaviour in Figure 14.

At low injection pressures, the aperture evolution is more impacted by the swelling than the effective stress in the fracture. It is interesting to observe the permeability evolution presents then a minimum before the permeability rebounds. Indeed, the rate of the swelling increment is decreasing since a Langmuir-like equation is considered. The rebound pressure can be estimated differentiating the analytical model and equalizing to zero. For the constant stiffness, the rebound pressure for the full rigid boundaries is given by

$$
p_{r b}=\sqrt{K_{m} \cdot \beta_{\varepsilon} \cdot V_{L} \cdot P_{L}}-P_{L}
$$

which is $4.06 \mathrm{MPa}$ in Figure 14. For the reservoir boundary conditions in Figure 15, it is

$$
p_{r b}=\sqrt{\frac{E_{m}}{3\left(1-v_{m}\right)} \cdot \beta_{\varepsilon} \cdot V_{L} \cdot P_{L}}-P_{L}
$$

which is $3.55 \mathrm{MPa}$ with the parameters used in Figure 14.

Finally, Figure 16 compares the constant fracture stiffness model for reservoir conditions with the Shi \& Durucan SD-model given by Equation (58) for the same conditions. In this equation, the stiffness $K_{p}$ is taken to be $K_{n} \cdot h_{0}$. The exponential law from Shi \& Durucan is close to the analytical solution presented above.

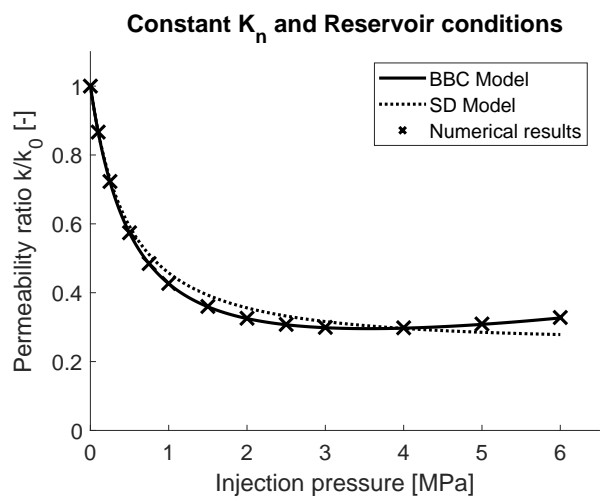

Figure 16: Constant fracture stiffness and reservoir boundary conditions: Comparison with the model from Shi \& Durucan (Equation 58 with $K_{p}=h_{0} \cdot K_{n}$ ). 


\begin{tabular}{llrl}
\hline Parameter & \multicolumn{3}{c}{ Value } \\
\hline Young's modulus & $E_{m}$ & 1.21 & $\mathrm{GPa}$ \\
Poisson's ratio & $\mathrm{V}_{m}$ & 0.16 & \\
Langmuir's volume & $V_{L}$ & 0.054 & $\mathrm{~m}^{3} / \mathrm{kg}$ \\
Langmuir's pressure & $P_{\varepsilon_{L}}$ & 1 & $\mathrm{MPa}$ \\
Swelling strain coefficient & $\beta_{\varepsilon}$ & 0.8 & $\mathrm{~kg} / \mathrm{m}^{3}$ \\
Matrix block width & $w_{0}$ & 0.003 & $\mathrm{~m}$ \\
Cleat aperture & $h_{0}$ & $18 \cdot 10^{-6}$ & $\mathrm{~m}$ \\
Cleat normal stiffness & $K_{n}^{0}$ & $1.66 \cdot 10^{6}$ & $\mathrm{MPa} \cdot \mathrm{m}^{-1}$ \\
& & & \\
\hline
\end{tabular}

Table 1: Model parameters.

\section{Numerical REV modelling}

The interest of the numerical model is that it can be used on complex geometries and it easily takes into account the anisotropy coming from the material and the boundary conditions. Moreover, the transient behaviour is also modelled. Figure 17 represents the geometry and the boundary conditions considered for the numerical modelling. A representative elementary volume (REV) made up of five blocks and two halfblocks arranged in three rows is meshed. The width of the blocks is $3 \mathrm{~mm}$. The axial stress applied for the reservoir boundary conditions is 4MPa. In both cases, the initial internal stress in the material is $4 \mathrm{MPa}$. The parameters used for the numerical modelling are the ones given in Table 1. In addition, a diffusion coefficient of $10^{-10} \mathrm{~m}^{2} / \mathrm{s}$ is considered. For this modelling, the gas pressure is increased in few hours to the wanted injection pressure at the end of every fracture on the boundaries (Figure 17).

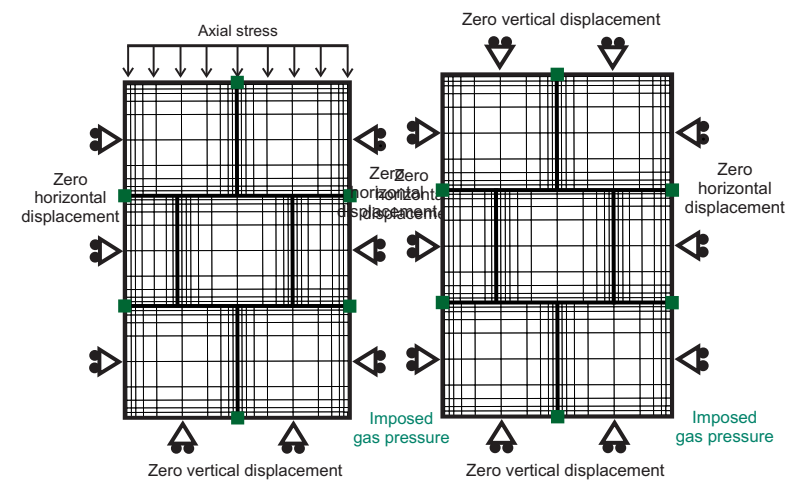

(a) Reservoir conditions.

(b) Full rigid boundaries.

Figure 17: REV geometry and boundary conditions considered for the modelling. 


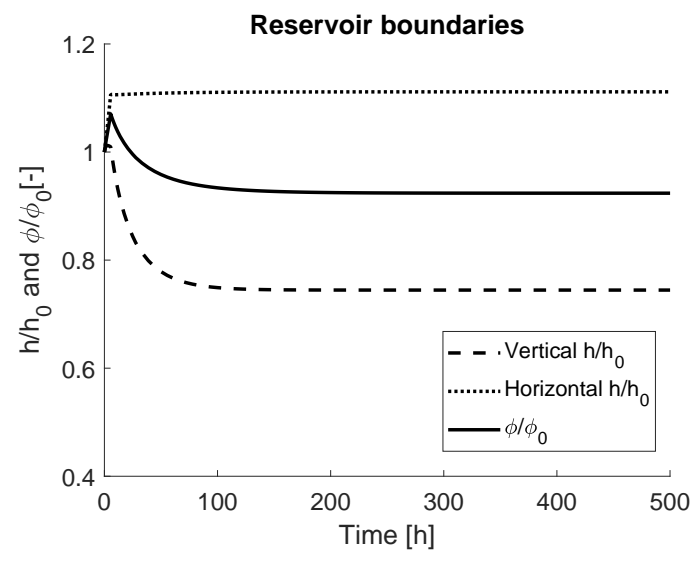

Figure 18: Vertical and horizontal fracture aperture evolution with time (injection pressure: 3MPa).

As the reservoir boundary conditions are anisotropic, the response of the horizontal fractures differs from the vertical fractures. Figure 18 presents the fracture aperture evolution with time of the vertical and horizontal fractures. The injection pressure was increased to $3 \mathrm{MPa}$ and then maintained constant. An initial opening of the fractures is observed horizontally and vertically during the loading phase, it is the effect of the effective stress decrease. Then, as the material is constrained horizontally, the vertical fractures are closing with the swelling. In contrast, the material is not constrained vertically and the horizontal fractures are opening. The global porosity evolution lies between the vertical and horizontal aperture evolutions.

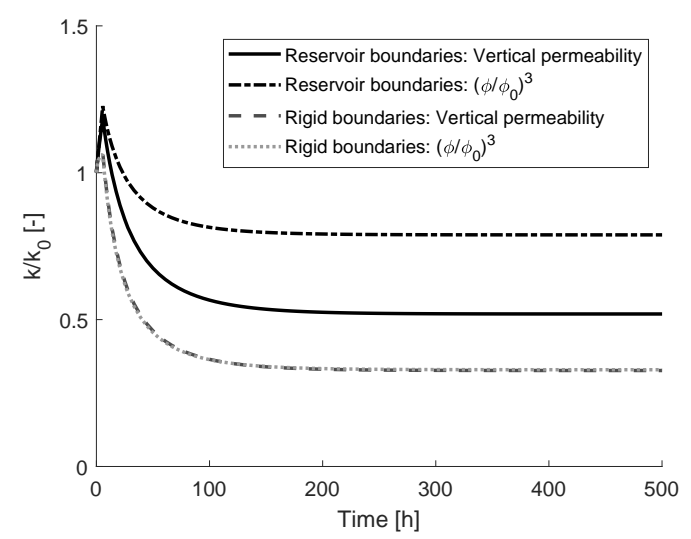

Figure 19: Equivalent vertical permeability evolution with time compared to the porosity model (injection pressure: $3 \mathrm{MPa}$ ).

From the curves in Figure 18, one can understand an analytical model based on the porosity evolution is not able to correctly represent the permeability evolution for both the vertical and horizontal fractures. Indeed, the porosity is a scalar variable while the 
permeability may be anisotropic, it is a tensor. Figure 19 represents the evolution of the vertical permeability of the REV. This permeability was computed using a weighted harmonic average following the path of the fluid in the vertical and horizontal fractures to flow from the bottom to the top of the REV. As expected, the permeability evolution based on the porosity evolution largely deviates when anisotropic boundaries are considered. However, in the case of full rigid boundaries, the vertical and horizontal apertures are closing in the same way and the porosity model perfectly fit the permeability evolution.

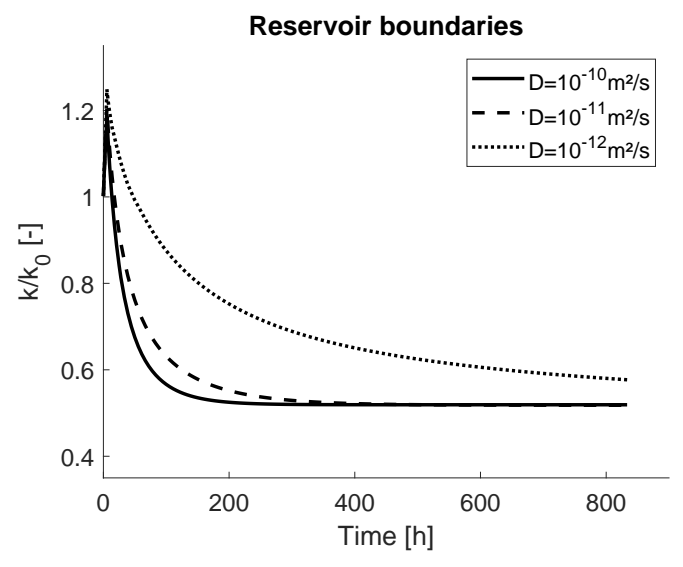

Figure 20: Equivalent vertical permeability evolution with time for different diffusion coefficients (injection pressure: $3 \mathrm{MPa}$ ).

The previous figures are presenting the evolution of the permeability evolution with time. This transient behaviour depends actually on the diffusion coefficient in the matrix. For lower values of this coefficient, the swelling occurs more slowly and the permeability decreases more slowly (Figure 20). It tends towards the same stabilized permeability value. 


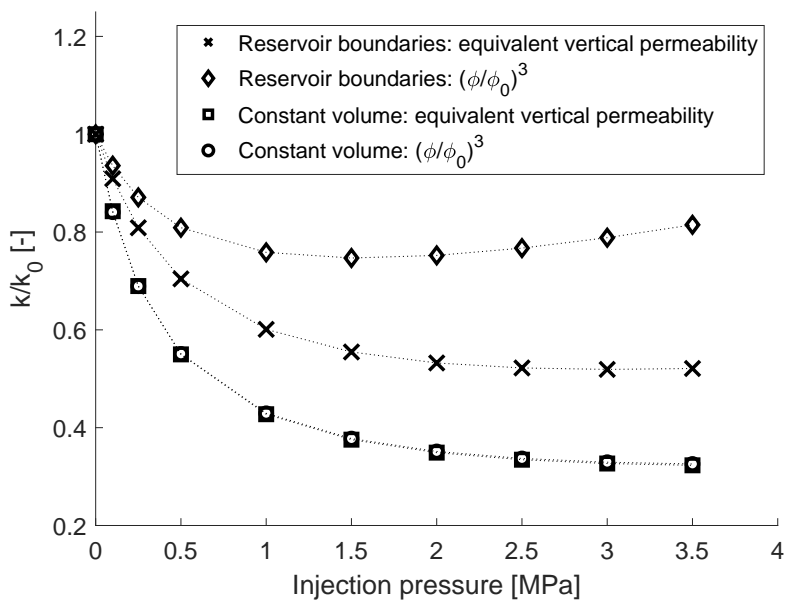

Figure 21: Permeability evolution with the pore pressure.

Figure 21 represents the (stabilized) permeability evolution with the pore pressure. Each result was obtained simulating the permeability evolution with the swelling for different injection pressures. The gas slippage phenomenon was not considered in these simulations in order to only highlight the effects of the swelling and the effective stress. As already noticed, the porosity model is not adequate to represent the permeability evolution in reservoir boundary conditions, it underestimates the decrease of the vertical permeability since the porosity integrates the opening of the horizontal fractures, what predicts a rebound pressure happening already at low pressure. It is therefore wiser to consider full rigid boundaries when applying a porosity model. The use of the numerical model avoids this problem for any geometry.

Note the computational cost of the direct modelling of the full microstructure would be highly expensive at the scale of the reservoir. However, the model presented in this paper is very useful for the modelling of the representative elementary volume which, using homogenization techniques, would define the macroscale behaviour of the material (Marinelli et al., 2016; Argilaga et al., 2016).

\section{Conclusion}

A hydro-mechanical model was developed at the scale of the coal constituents to distinguish the hydro-mechanical behaviour of the matrix and the cleats. For the matrix, a simple isotropic linear elastic law is used for the mechanical behaviour and a diffusion law is considered for the gas flow. The swelling/shrinkage of the matrix is linked to its gas content and, depending on the boundary conditions, this sorptioninduced strain may impact the fracture aperture. It is considered that the normal stiffness evolves with the fracture closure. This closure modifies the permeability of the fracture and impacts the fluid flow in the fracture. As a multi-phase flow may be en- 
countered in the cleats in the context of coalbed methane recovery, a full model was presented for unsaturated conditions. The Langmuir's isotherm is innovatively included in the gas transfer between the cleats and the matrix.

The implementation of the model was validated by comparison between some stabilized permeability values obtained numerically for different $\mathrm{CO}_{2}$ injection pressures and the analytical solution of the problem. The analytical solution was developed for different boundary conditions and for different expressions of the normal stiffness of the fracture. The constant stiffness case is also compared to a classical exponential model derived in the literature from the bulk deformation of the material. Finally, the model was used for the modelling of a representative elementary volume made up few blocks. This REV combines horizontal and vertical fractures that impacts differently the permeability evolution if anisotropic boundaries conditions are encountered. In this case, it was shown that models based on the porosity evolution largely deviates from the direct fracture aperture model, such it is supposed to be in the reservoir. This numerical model at the scale of the matrix and the cleats should be integrated into a multi-scale approach to be able to model gas flows at the reservoir scale.

\section{Acknowledgements}

These researches were supported by the FNRS-FRIA and the WBI.

\section{Appendix A. Gas slippage phenomenon}

The problem of gas slippage in capillary tubes and its effect on permeability in porous media was first addressed by Klinkenberg (Klinkenberg et al., 1941). A model for the flow between two parallel plates is derived. Kundt and Warburg showed that the velocity at the wall is proportional to the velocity gradient at the wall (Kundt and Warburg, 1875):

$$
\left.v_{1}\left(\frac{h_{b}}{2}\right)=-c \bar{l} \frac{\mathrm{d} v_{1}}{\mathrm{~d} x_{2}}\right]_{x_{2}=\frac{h_{b}}{2}}
$$

where $c$ is a constant with a value slightly less than 1 according to Kundt and Warburg and $\bar{l}$ is the mean free path of the gas molecules at the mean pressure. The velocity gradient is obtained by integrating equation (30), it gives:

$$
\frac{\mathrm{d} v_{1}}{\mathrm{~d} x_{2}}=\frac{x_{2}}{\mu} \frac{\mathrm{d} p}{\mathrm{~d} x_{1}}+C
$$

To ensure an extremum value in the center $\left(x_{2}=0\right)$, the constant $C$ must be zero. Thence, the velocity at the walls is:

$$
v_{1}\left(\frac{h_{b}}{2}\right)=-\frac{c \bar{l} h_{b}}{2 \mu} \frac{\mathrm{d} p}{\mathrm{~d} x_{1}}
$$


These boundary conditions allows to find the following velocity profile:

$$
v_{1}\left(x_{2}\right)=-\frac{1}{2 \mu}\left(c \bar{l} h_{b}+\left(\frac{h_{b}}{2}\right)^{2}-x_{2}^{2}\right) \frac{\mathrm{d} p}{\mathrm{~d} x_{1}}
$$

Averaging this velocity over the aperture, we find:

$$
q=\left\langle v_{1}\right\rangle=\frac{1}{h_{b}} \int_{-\frac{h_{b}}{2}}^{\frac{h}{2}} v_{1} d x_{2}=-\frac{h_{b}^{2}}{12}\left(1+\frac{6 c \bar{l}}{h_{b}}\right) \cdot \frac{1}{\mu} \frac{\mathrm{d} p}{\mathrm{~d} x_{1}}
$$

In comparison with Equation (33), one can observe that the permeability is increased of a dimensionless correction factor $f_{c}$ given by:

$$
f_{c}=\left(1+\frac{6 c l}{h_{b}}\right)=\left(1+\frac{b}{\bar{p}}\right) \quad \text { with } \quad \frac{b}{\bar{p}}=\frac{6 c \bar{l}}{h_{b}}=6 c \kappa_{n},
$$

where $c \approx 1$ and $b$ is the Klinkenberg number. For a capillary tube, it has been found that $\frac{b}{\bar{p}}=\frac{4 c \bar{l}}{r}$ with $r$ the effective pore radius (Klinkenberg et al., 1941). Note the gas permeability is a function of the mean free path $l$ of the gas molecules, it depends therefore on the pressure, the temperature and the nature of the gas.

\section{Appendix B. Unsaturated flow model}

Generally, gas and water flows are simultaneously encountered in the cleats and it is therefore necessary to extend the model to unsaturated conditions. In this purpose, we introduce the widely-used concept of relative permeability $k_{r}$ as a measure of the reduction in permeability to a given phase that occurs between partially and fully saturated conditions. In petroleum engineering, the relative permeabilities of the wetting phase $\left(k_{r w}\right)$ and the non-wetting phase $\left(k_{r n w}\right)$ in porous media are often expressed as power law functions of saturation $\left(S_{r}\right)$, known as Corey functions (Corey, 1954). Contrary to a macroscopic approach, it must be assumed the two phases are simultaneously flowing in a single cleat. Logically, the space occupied by one phase is not available for the flow of the other phase. Based on the work of Romm, a simple model consists to equalize the relative permeabilities to the phases saturations, it is known as the $\mathrm{X}$ curves (Romm, 1966):

$$
k_{r w}=S_{r} \quad \text { (B.1) } \quad k_{r g}=S_{g}=1-S_{r}
$$

As can be noticed, the sum of $k_{r w}$ and $k_{r g}$ equals 1 in the $\mathrm{X}$ model, which means each phase flows in its own path without impeding the flow of the other, the interference between the phases is not considered.

Through a real fracture, the $\mathrm{X}$ model may no longer be valid if the interference is significant. In order to characterize the deviation from the $\mathrm{X}$ model, Chen and Horne proposed the following expressions (Chen et al., 2004): 


$$
k_{r w}=\frac{S_{r}}{\tau_{c, w}} \quad \text { (B.3) } \quad k_{r g}=\frac{1-S_{r}}{\tau_{c, g}}
$$

where $\tau_{c, w}$ and $\tau_{c, g}$ are the channel tortuosities of the two phases. These coefficients, varying between 1 and the infinity, quantify the shear stress at the interface between the two fluids. Chen and Horne evaluate the tortuosity coefficients analysing the structure flow by digital image processing techniques. Unfortunately, a model that predicts the flow structure in a fracture does not exist. It is therefore impossible to evaluate $\tau_{c, w}$ and $\tau_{c, g}$ without resorting to experiments. However, assuming a simple flow structure, it is possible to derive a model which takes into account the interference between the two phases through viscous considerations. Yuster used co-axial flows in a single circular cylinder to infer a viscosity ratio dependence of the relative permeabilities (Yuster et al., 1951). This viscous coupling model can also be applied to a two-phase flow between two plates (Fourar et al., 1998). We consider two fluids flowing simultaneously with a planar interface. Water is the wetting fluid and is therefore in contact with the walls, and gas (non-wetting) flows in between (Figure B.22).

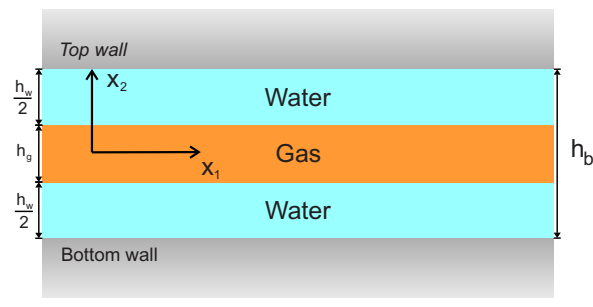

Figure B.22: Gas flow in between of water flows in a fracture space.

The viscous coupling between the fluids is derived by integrating Stokes' equation in each stratum. The velocity of each phase is the same at the interface, the shear force on the two phases is therefore the same at the interface:

$$
\begin{gathered}
L \cdot w \cdot \mu_{w}\left(\frac{\mathrm{d} v_{1}}{\mathrm{~d} x_{2}}\right)_{w}=L \cdot w \cdot \mu_{g}\left(\frac{\mathrm{d} v_{1}}{\mathrm{~d} x_{2}}\right)_{g} \\
\Rightarrow \mu_{w}\left(\frac{\mathrm{d} v_{1}}{\mathrm{~d} x_{2}}\right)_{w}=\mu_{g}\left(\frac{\mathrm{d} v_{1}}{\mathrm{~d} x_{2}}\right)_{g}
\end{gathered}
$$

Equations of the form given by Equation (A.2) for the two phases at the interface $\left(x_{2}=\frac{h_{g}}{2}\right)$ are:

$$
\left(\frac{\mathrm{d} v_{1}}{\mathrm{~d} x_{2}}\right)_{w}=\frac{h_{g}}{2 \mu_{w}} \frac{\mathrm{d} p}{\mathrm{~d} x_{1}}+C_{w}
$$




$$
\left(\frac{\mathrm{d} v_{1}}{\mathrm{~d} x_{2}}\right)_{g}=\frac{h_{g}}{2 \mu_{g}} \frac{\mathrm{d} p}{\mathrm{~d} x_{1}}+C_{g}
$$

Due to symmetry, the derivative of the velocity equals zero at the center of the fracture $\left(x_{2}=0\right)$; it means that $C_{g}=0$. Moreover, to satisfy Equation (B.5), we have:

$$
\mu_{w} \cdot C_{w}=\mu_{g} \cdot C_{g}
$$

It follows that $C_{w}=0$. Therefore, the generalized expression for the velocity gradient perpendicular to the direction of flow is:

$$
\frac{\mathrm{d} v_{1}}{\mathrm{~d} x_{2}}=\frac{x_{2}}{\mu} \frac{\mathrm{d} p}{\mathrm{~d} x_{1}}
$$

Integrating Equation (B.9) to give the velocity profile perpendicular to the direction of flow leads to:

$$
v_{1}=\frac{x_{2}^{2}}{2 \mu} \frac{\mathrm{d} p}{\mathrm{~d} x_{1}}+Z
$$

Applying this to the water phase, when $x_{2}=\frac{h_{b}}{2}$, then $v_{1}=0$ and Equation (31) is found:

$$
v_{1_{w}}=-\frac{1}{2 \mu_{w}}\left(\left(\frac{h_{b}}{2}\right)^{2}-x_{2}^{2}\right) \frac{\mathrm{d} p_{w}}{\mathrm{~d} x_{1}}
$$

Thence, the velocity at the interface is:

$$
v_{1 i}=-\frac{1}{2 \mu_{w}}\left(\left(\frac{h_{b}}{2}\right)^{2}-\left(\frac{h_{g}}{2}\right)^{2}\right) \frac{\mathrm{d} p}{\mathrm{~d} x_{1}}
$$

For the gas phase, the velocity distribution is:

$$
v_{1 g}=\frac{x_{2}^{2}}{2 \mu_{g}} \frac{\mathrm{d} p_{g}}{\mathrm{~d} x_{1}}+Z_{g}
$$

And at the interface:

$$
v_{1 i}=\frac{1}{2 \mu_{g}}\left(\frac{h_{g}}{2}\right)^{2} \frac{\mathrm{d} p}{\mathrm{~d} x_{1}}+Z_{g}
$$

Since the velocity of the two phases at the interface has been assumed to be the same, then Equations (B.12) and (B.14) may be equated to find $Z_{g}$. Thence, from Equation (B.13), we obtain the following velocity profile in the gas phase:

$$
v_{1 g}=-\frac{1}{2}\left[\frac{1}{\mu_{w}}\left(\left(\frac{h_{b}}{2}\right)^{2}-\left(\frac{h_{g}}{2}\right)^{2}\right)+\frac{1}{\mu_{g}}\left(\left(\frac{h_{g}}{2}\right)^{2}-x_{2}^{2}\right)\right] \frac{\mathrm{d} p_{g}}{\mathrm{~d} x_{1}}
$$

Equations (B.11) and (B.15) give the velocity profile in the water and gas phases re- 
spectively. To derive the relative permeabilities, it is necessary to calculate the average velocity of each phase over the aperture. For the liquid phase, it gives:

$$
\begin{aligned}
q_{l_{L}} & =\frac{2}{h_{b}} \int_{\frac{h_{g}}{2}}^{\frac{h_{b}}{2}} v_{1 w} d x_{2} \\
& =-\frac{h_{b}^{2}}{12}\left(1-\frac{3}{2} \frac{h_{g}}{h_{b}}+\frac{1}{2}\left(\frac{h_{g}}{h_{b}}\right)^{3}\right) \frac{1}{\mu_{w}} \frac{\mathrm{d} p_{w}}{\mathrm{~d} x_{1}}
\end{aligned}
$$

Knowing that $\frac{h_{g}}{h_{b}}=\frac{h_{b}-h_{w}}{h_{b}}=1-S_{r}$, we have

$$
q_{l L}=-\frac{h_{b}^{2}}{12}\left(\frac{S_{r}^{2}}{2}\left(3-S_{r}\right)\right) \frac{1}{\mu_{w}} \frac{\mathrm{d} p_{w}}{\mathrm{~d} x_{1}}
$$

And for the gas phase, we have:

$$
\begin{aligned}
q_{g_{L}} & =\frac{2}{h_{b}} \int_{0}^{\frac{h_{g}}{2}} v_{1 g} d x_{2} \\
& =-\frac{h_{b}^{2}}{12}\left(\frac{\mu_{g}}{\mu_{w}} \frac{3}{2}\left(\frac{h_{g}}{h_{b}}\right)\left(1-\frac{h_{g}}{h_{b}}\right)^{2}+\left(\frac{h_{g}}{h_{b}}\right)^{3}\right) \frac{1}{\mu_{g}} \frac{\mathrm{d} p_{g}}{\mathrm{~d} x_{1}} \\
& =-\frac{h_{b}^{2}}{12}\left(\frac{3}{2} \mu_{r} S_{r}\left(1-S_{r}\right)\left(2-S_{r}\right)+\left(1-S_{r}\right)^{3}\right) \frac{1}{\mu_{g}} \frac{\mathrm{d} p_{g}}{\mathrm{~d} x_{1}}
\end{aligned}
$$

where $\mu_{r}=\frac{\mu_{g}}{\mu_{w}}$ is the viscosity ratio. So, the longitudinal flows are

$$
q_{l_{L}}=-k_{r w} \frac{h_{b}^{2}}{12} \frac{1}{\mu_{w}} \frac{\partial p_{w}}{\partial x_{1}}
$$

and

$$
q_{g_{L}}=-k_{r g} \frac{h_{b}^{2}}{12} \frac{1}{\mu_{g}} \frac{\partial p_{g}}{\partial x_{1}}
$$

with the following relative permeabilities:

$$
\begin{gathered}
k_{r w}=\frac{S_{r}^{2}}{2}\left(3-S_{r}\right) \\
k_{r g}=\left(1-S_{r}\right)^{3}+\frac{3}{2} \mu_{r} S_{r}\left(1-S_{r}\right)\left(2-S_{r}\right)
\end{gathered}
$$

Note if $\mu_{r}$ is larger than one, the relative permeability of the non-wetting phase is also larger than unity (lubrication effect). For $\mu_{r}$ equal to one, the sum of the $k_{r}$ is also equal to one; however, $k_{r}$ is not equal to saturation as for the $\mathrm{X}$ model. In fact, for gas and water two-phase flows, the viscosity ratio is largely lower than one and the second term on the right-hand side of Equation (B.22) is negligible. 
The relative permeability is not equal to the saturation but it is however a function of this variable. A retention curve is therefore required to derive the saturation degree from the primary unknowns of the problem, the water and gas pressures. The definition of a retention curve actually implies a distribution in the size of the fractures (or capillary tubes). With a macroscopic approach, depending on the capillary pressure, fractures are filled with water under a certain size and filled with gas above this size. At the microscale, we are interested in developing a capillary pressure model taking into account a multiphase flow in a single fracture. For this purpose, we take advantage of the natural irregular shape of the fracture wall. Realizing many natural structures are constituted of irregular objects appearing in a similar way when observed at different scales, a fractal technique (Mandelbrot and Pignoni, 1983) is applied to describe the geometry of the fracture wall.

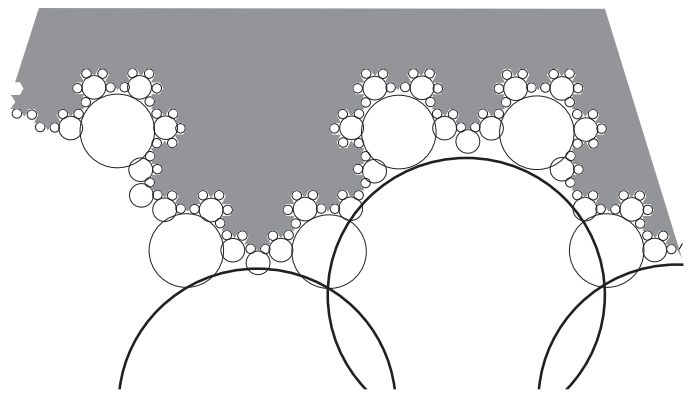

Figure B.23: A fracture wall seen as a fractal object with a distribution of open capillary tubes.

Assuming the fracture wall is a fractal object, the space between two walls can be filled by a fractal distribution of capillary tubes with smaller tubes at the walls and larger tubes in the middle (Figure B.23). Considering a capillary tube model, the capillary pressure can be expressed as:

$$
p_{c}=\frac{2 \sigma \cos \theta}{r}
$$

where $p_{c}$ is the capillary pressure, $\sigma$ is the surface tension and $\theta$ is the contact angle. It is assumed that capillary effects are also effective in open capillary tubes.

Mathematically, the number $N$ of units whose radius is larger than $r$ is represented by a power-law function (Kewen et al., 2004):

$$
N(r)=a \cdot r^{-D_{f}}
$$

where $D_{f}$ is the fractal dimension and $a$ a constant of proportionality. Defining the volume of the unit equal to $\pi r^{2} l$, the number of the units invaded by the non-wetting 
phase is

$$
N(r)=\frac{\Omega_{g}}{\pi r^{2} l}
$$

Combining Equations (B.24) and (B.25):

$$
\Omega_{g}=a \pi l r^{\left(2-D_{f}\right)}
$$

Substituting Equation (B.23) in Equation (B.26), the saturation of the non-wetting phase is then:

$$
S_{r_{g}}=\frac{\Omega_{g}}{\Omega_{v}}=b \cdot p_{c}^{-\left(2-D_{f}\right)}
$$

with $b$ a constant of proportionality. Differentiating Equation (B.27):

$$
\frac{d S_{r_{g}}}{d p_{c}}=c \cdot p_{c}^{-\left(3-D_{f}\right)}
$$

with $c$ a constant of proportionality. Then, assuming $p_{c}$ tends to $p_{e}$ when $S_{r_{g}}=0$ and $p_{c}$ approaches infinity when $S_{r_{g}}=1-S_{r, \text { res }}-S_{r_{g}, \text { res }}$, by integrating Equation (B.28):

$$
\begin{aligned}
\frac{\int_{0}^{1-S_{r}-S_{r_{g}, \text { res }}} d S_{r}}{\int_{0}^{1-S_{r_{r} \text { res }}-S_{r_{g}, \text { res }}} d S_{r}} & =\frac{\int_{p_{e}}^{p_{c}} p_{c}^{-\left(3-D_{f}\right)}}{\int_{p_{e}}^{+\infty} p_{c}^{-\left(3-D_{f}\right)}} \\
\frac{1-S_{r}-S_{r_{g}, \text { res }}}{1-S_{r, \text { res }}-S_{r_{g}, \text { res }}} & =\frac{p_{c}^{-\left(2-D_{f}\right)}-p_{e}^{-\left(2-D_{f}\right)}}{p_{e}^{-\left(2-D_{f}\right)}} \\
& =1-\left(\frac{p_{c}}{p_{e}}\right)^{-\left(2-D_{f}\right)}
\end{aligned}
$$

Equation (B.31) is obtained considering the fractal dimension is lower than 2. Reducing this equation leads to:

$$
p_{c}=p_{e} \cdot\left(S_{r}^{*}\right)^{-\frac{1}{2-D_{f}}}
$$

where $S_{r}^{*}$ is the normalized saturation. As can be observed, Equation (B.32) is the same form as the Brooks-Corey equation:

$$
p_{c}=p_{e} \cdot\left(S_{r}^{*}\right)^{-\frac{1}{\lambda}}
$$

where $\lambda$ is an index representing the size distribution of the capillary tubes.

\section{References}

Argilaga, A., Papachristos, E., Caillerie, D., and Dal Pont, S. (2016). Homogenization of a cracked saturated porous medium: Theoretical aspects and numerical imple- 
mentation. International Journal of Solids and Structures, 94:222-237.

Bandis, S., Lumsden, A., and Barton, N. (1983). Fundamentals of rock joint deformation. In International Journal of Rock Mechanics and Mining Sciences \& Geomechanics Abstracts, volume 20, pages 249-268. Elsevier.

Barenblatt, G., Zheltov, I. P., and Kochina, I. (1960). Basic concepts in the theory of seepage of homogeneous liquids in fissured rocks [strata]. Journal of applied mathematics and mechanics, 24(5):1286-1303.

Barton, N., Bandis, S., and Bakhtar, K. (1985). Strength, deformation and conductivity coupling of rock joints. In International Journal of Rock Mechanics and Mining Sciences \& Geomechanics Abstracts, volume 22, pages 121-140. Elsevier.

Berkowitz, N. (1985). The chemistry of coal.

Bertrand, F., Cerfontaine, B., and Collin, F. (2017). A fully coupled hydro-mechanical model for the modeling of coalbed methane recovery. Journal of Natural Gas Science and Engineering, 46:307-325.

Cerfontaine, B., Dieudonné, A.-C., Radu, J.-P., Collin, F., and Charlier, R. (2015). 3d zero-thickness coupled interface finite element: formulation and application. Computers and Geotechnics, 69:124-140.

Charlier, R. (1987). Approche unifiée de quelques problèmes non linéaires de mécanique des milieux continus par la méthode des éléments finis (grandes déformations des métaux et des sols, contact unilatéral de solides, conduction thermique et écoulements en milieu poreux). $\mathrm{PhD}$ thesis, Université de Liège.

Chen, C.-Y., Horne, R. N., and Fourar, M. (2004). Experimental study of liquid-gas flow structure effects on relative permeabilities in a fracture. Water Resources Research, 40(8).

Collin, F., Li, X.-L., Radu, J.-P., and Charlier, R. (2002). Thermo-hydro-mechanical coupling in clay barriers. Engineering Geology, 64(2):179-193.

Connell, L. D., Lu, M., and Pan, Z. (2010). An analytical coal permeability model for tri-axial strain and stress conditions. International Journal of Coal Geology, 84(2):103-114.

Corey, A. T. (1954). The interrelation between gas and oil relative permeabilities. Producers monthly, 19(1):38-41.

Cui, X. and Bustin, R. M. (2005). Volumetric strain associated with methane desorption and its impact on coalbed gas production from deep coal seams. Aapg Bulletin, 89(9):1181-1202.

Cussler, E. L. (2009). Diffusion: mass transfer in fluid systems. Cambridge university press. 
Darcy, H. (1856). Les fontaines publiques de la ville de Dijon. Librairie des Corps Impériaux des Ponts et Chaussées et des Mines.

Fick, A. (1855). Ueber diffusion. Annalen der Physik, 170(1):59-86.

Fourar, M., Lenormand, R., et al. (1998). A viscous coupling model for relative permeabilities in fractures. In SPE Annual Technical Conference and Exhibition. Society of Petroleum Engineers.

Gens, A., Carol, I., and Alonso, E. (1990). A constitutive model for rock joints formulation and numerical implementation. Computers and Geotechnics, 9(1-2):3-20.

Gilman, A. and Beckie, R. (2000). Flow of coal-bed methane to a gallery. Transport in porous media, $41(1): 1-16$.

Goodman, R. E. (1976). Methods of geological engineering in discontinuous rocks.

Gray, I. et al. (1987). Reservoir engineering in coal seams: Part 1-the physical process of gas storage and movement in coal seams. SPE Reservoir Engineering, 2(01):2834.

Gustafsson, B. and Vasil'ev, A. (2006). Conformal and potential analysis in Hele-Shaw cells. Springer Science \& Business Media.

Habraken, A.-M. (1989). Contribution à la modélisation du formage des métaux par la méthode des éléments finis. $\mathrm{PhD}$ thesis, Universté de Liège.

Harpalani, S. and Chen, G. (1997). Influence of gas production induced volumetric strain on permeability of coal. Geotechnical \& Geological Engineering, 15(4):303325 .

Intergovernmental Panel on Climate Change - IPCC (2015). Climate change 2014: Mitigation of climate change, volume 3. Cambridge University Press.

Ismail, A. F., Khulbe, K. C., and Matsuura, T. (2015). Gas separation membranes, volume 7. Springer.

Karacan, C. Ö. (2003). Heterogeneous sorption and swelling in a confined and stressed coal during co2 injection. Energy \& Fuels, 17(6):1595-1608.

Kewen, L. et al. (2004). Theoretical development of the brooks-corey capillary pressure model from fractal modeling of porous media. In SPE/DOE Symposium on Improved Oil Recovery. Society of Petroleum Engineers.

Klinkenberg, L. et al. (1941). The permeability of porous media to liquids and gases. In Drilling and production practice. American Petroleum Institute.

Kundt, A. and Warburg, E. (1875). Ueber reibung und wärmeleitung verdünnter gase. Annalen der Physik, 232(10):177-211.

Langmuir, I. (1918). The adsorption of gases on plane surfaces of glass, mica and platinum. Journal of the American Chemical society, 40(9):1361-1403. 
Lu, S., Cheng, Y., and Li, W. (2016). Model development and analysis of the evolution of coal permeability under different boundary conditions. Journal of Natural Gas Science and Engineering, 31:129-138.

Mandelbrot, B. B. and Pignoni, R. (1983). The fractal geometry of nature.

Marinelli, F., Van den Eijnden, A., Sieffert, Y., Chambon, R., and Collin, F. (2016). Modeling of granular solids with computational homogenization: Comparison with biot's theory. Finite Elements in Analysis and Design, 119:45-62.

Olsson, R. and Barton, N. (2001). An improved model for hydromechanical coupling during shearing of rock joints. International Journal of Rock Mechanics and Mining Sciences, 38(3):317-329.

Pan, Z. and Connell, L. D. (2007). A theoretical model for gas adsorption-induced coal swelling. International Journal of Coal Geology, 69(4):243-252.

Pan, Z. and Connell, L. D. (2012). Modelling permeability for coal reservoirs: a review of analytical models and testing data. International Journal of Coal Geology, 92:144.

Peng, Y., Liu, J., Pan, Z., Connell, L. D., Chen, Z., and Qu, H. (2017). Impact of coal matrix strains on the evolution of permeability. Fuel, 189:270-283.

Reiss, L. H. (1980). The reservoir engineering aspects of fractured formations, volume 3. Editions Technip.

Romm, E. S. (1966). Fluid flow in fractured rocks (in Russian). Nedra Publishing House.

Schwerer, F., Pavone, A., et al. (1984). Effect of pressure-dependent permeability on well-test analyses and long-term production of methane from coal seams. In SPE Unconventional Gas Recovery Symposium. Society of Petroleum Engineers.

Seidle, J., Jeansonne, M., Erickson, D., et al. (1992). Application of matchstick geometry to stress dependent permeability in coals. In SPE rocky mountain regional meeting. Society of Petroleum Engineers.

Shi, J. and Durucan, S. (2003). A bidisperse pore diffusion model for methane displacement desorption in coal by $\mathrm{CO}_{2}$ injection. Fuel, 82(10):1219-1229.

Shi, J. and Durucan, S. (2004). Drawdown induced changes in permeability of coalbeds: a new interpretation of the reservoir response to primary recovery. Transport in porous media, 56(1):1-16.

Shi, J.-Q., Pan, Z., and Durucan, S. (2014). Analytical models for coal permeability changes during coalbed methane recovery: Model comparison and performance evaluation. International Journal of Coal Geology, 136:17-24. 
Somerton, W. H., Soylemezoglu, I., and Dudley, R. (1975). Effect of stress on permeability of coal. In International journal of rock mechanics and mining sciences \& geomechanics abstracts, volume 12, pages 129-145. Elsevier.

Warren, J., Root, P. J., et al. (1963). The behavior of naturally fractured reservoirs. Society of Petroleum Engineers Journal, 3(03):245-255.

White, C. M., Smith, D. H., Jones, K. L., Goodman, A. L., Jikich, S. A., LaCount, R. B., DuBose, S. B., Ozdemir, E., Morsi, B. I., and Schroeder, K. T. (2005). Sequestration of carbon dioxide in coal with enhanced coalbed methane recovery a review. Energy \& Fuels, 19(3):659-724.

Witherspoon, P. A., Wang, J., Iwai, K., and Gale, J. (1980). Validity of cubic law for fluid flow in a deformable rock fracture. Water resources research, 16(6):1016-1024.

Wu, K., Li, X., Wang, C., Yu, W., and Chen, Z. (2015). Model for surface diffusion of adsorbed gas in nanopores of shale gas reservoirs. Industrial \& Engineering Chemistry Research, 54(12):3225-3236.

Yuster, S. et al. (1951). Theoretical considerations of multiphase flow in idealized capillary systems. In Proceedings of the 3rd World Petroleum Congress, Section II, The Hague, volume 2, pages 437-445.

Zienkiewicz, O. C. and Taylor, R. L. (2000). The finite element method: solid mechanics, volume 2. Butterworth-heinemann.

Zimmerman, R. W., Somerton, W. H., and King, M. S. (1986). Compressibility of porous rocks. Journal of Geophysical Research: Solid Earth, 91(B12):1276512777. 